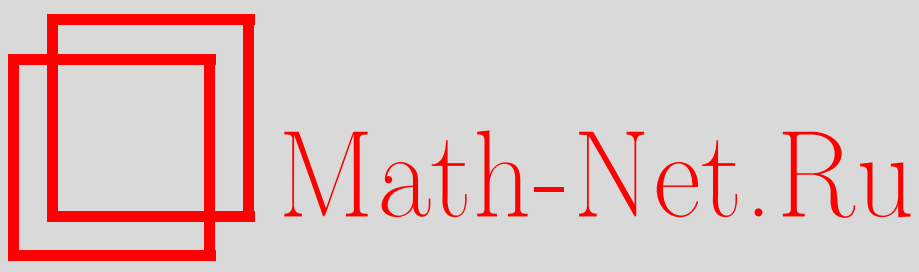

О. В. Бесов, Теорема вложения Соболева для области с нерегулярной границей, матем. сб., 2001, том 192, номер 3, 3-26

DOI: https://doi.org/10.4213/sm548

Использование Общероссийского математического портала Math-Net.Ru подразумевает, что вы прочитали и согласны с пользовательским соглашением http: //www. mathnet.ru/rus/agreement

Параметры загрузки:

IP : 35.174 .16 .151

26 апреля 2023 г., 11:29:20 
УДК 517.51

\title{
O.В. Бесов \\ Теорема вложения Соболева для области с нерегулярной границей
}

\begin{abstract}
В теореме вложения Соболева $W_{p}^{s}(G) \subset L_{q}(G)$ допустимые соотношения между параметрами гладкости и суммируемости определяются геометрическими свойствами области $G$. В работе эта теорема и соответствующие оценки слабого типа устанавливаются для областей с нерегулярной границей и в случае весовых пространств $L_{p}, L_{q}$.

Библиография: 15 названий.
\end{abstract}

Известная теорема вложения Соболева

$$
W_{p}^{s}(G) \subset L_{q}(G), \quad s \in \mathbb{N}, \quad 1<p<q<\infty
$$

установлена С.Л. Соболевым в 1938 г. (см. [1]) для области $G \subset \mathbb{R}^{n}$ с условием конуса при $s-n / p+n / q \geqslant 0$. Последнее соотношение (определяюшее максимально возможное значение $q$ в теореме $(1))$ является и необходимьм условием вложения. Результат С. Л. Соболева был перенесен на области более общего вида: области классов $J_{\frac{n-1}{n}}, I_{p, \frac{1}{p}-\frac{1}{n}}$ (В.Г. Мазья, 1960 г., 1975 г., см. [2]), области с условием Джона (Ю.Г. Решетняк [3], [4]), области с условием гибкого конуса (О.В. Бесов, 1983 г., см. [5]).

В случае области $G$ с нерегулярной границей (например, имеющей вид внешнего пика в окрестности некоторой точки границы) вложение оказывается верньм лишь при более сильных условиях, связывающих $s, p, q$ и зависящих от геометрических свойств границы $G$. В.Г. Мазья выделил классы областей $J_{\alpha}(1960$ г.), $I_{p, \alpha}(1975$ г.), для которых установил соответственно при $p=1$ и $p>1$ теорему вложения (1) при $s=1$ и максимально возможном $q(=1 / \alpha)$, см. [2]. Условия принадлежности области $G$ классам $J_{\alpha}, I_{p, \alpha}$, формулируемые в терминах изопериметрических или емкостных неравенств, трудно проверяемы. И. Г. Глобенко в 1962 г. [6] для областей с условием вырожденного конуса (степенное вырождение) установил теорему вложения (1) с максимально возможным $q$ при $s=1$. Его доказательство при $s=2,3, \ldots$ вызывает сомнение, см. работу Д. А. Лабутина [7], в которой приведено доказательство вложения (1) при $s \in \mathbb{N}$ с максимально возможньм значением $q$ для областей более общего вида, имеющих, в частности, внешние пики степенного типа. Ранее Смит и Стегенга [8] для области $G$ с $\sigma$-условием Джона ( $\sigma$-John domain), $\sigma>1$, установили при $q=p(s=1)$ неравенство Соболева-Пуанкаре. Хайлаш и Коскела [9] для области с $\sigma$-условием Джона $(\sigma \geqslant 1)$

Работа выполнена при финансовой поддержке Российского фонда фундаментальных исследований (грант № 99-01-00868), Российской программы поддержки ведущих научных школ (грант № 00-15-96047) и фонда INTAS (грант № 99-01080).

(C) О.В. Бесов 2001 
установили неравенство Соболева-Пуанкаре $(s=1)$ со значением $q$, близким к максимально возможному, а при $p=1$ - с максимально возможным. Неравенство Соболева-Пуанкаре $(s=1)$ для области с $\sigma$-условием Джона $(\sigma>1)$ при $p>1$ и максимально возможном $q$ установили Килпелайнен и Малы [10] в качестве следствия из более общего результата в отношении геометрических свойств области $G$. В [9], [10] содержатся также обобщения на случай весовых $L_{p^{-}}, L_{q^{-}}$пространств (вес - степень расстояния до границы области).

Теорема вложения $W_{p}^{1}(G) \rightarrow L_{q}(G)$ с максимальным показателем $q$ для гёльдеровых областей $G$ установлена Д.А. Лабутиным [11]. Отметим еще работу Стредулинского [12], в которой упомянутые теоремы вложения В.Г. Мазьи распространены на весовые пространства Соболева.

Автор признателен Д. А. Лабутину, привлекшему его внимание к работам [10], [13] и к вопросу обобшения результатов на случай производных порядка $s$ выше первого.

В настоящей работе устанавливается теорема вложения (1) при $s \in \mathbb{N}$ для областей $G$ некоторого класса и, в частности, для областей $G$ с $\sigma$-условием Джона $(\sigma>1)$. При $s=1$ предлагаемый метод позволяет несколько расширить (сравнительно с [10]) класс областей, для которых справедливо неравенство Соболева-Пуанкаре. Результаты обобщаются и на случаи вложений пространств с весовьми нормами с достаточно общими весами.

Везде далее $\delta \in(0,1)$ достаточно мало; область $G \subset \mathbb{R}^{n} ; G_{\delta}=\{x \in G$ : $\operatorname{dist}(x, \partial G)>\delta / 2\} ; v, w: G \rightarrow(0, \infty), u: G_{\delta} \rightarrow(0, \infty) ; u, v, w$ локально суммируемы; для измеримого по Лебегу множества $E \subset \mathbb{R}^{n}|E|_{w}=\int_{E \cap G} w(x) d x$ и при $E \subset G, 1 \leqslant p<\infty,\|f\|_{p, E}=\left\|f\left|L_{p}(E)\|\| f,\right| L_{p, v}(E)\right\|=\left\|f v^{\frac{1}{p}} \mid L_{p}(E)\right\| ;$

$$
1 \leqslant p<q<\infty, \quad 1 \leqslant r \leqslant q, \quad s \in \mathbb{N}, \quad s-\frac{n}{p}+\frac{n}{q} \geqslant 0 .
$$

Цель работы - нахождение условий справедливости для области $G$ с нерегулярной границей оценки

$$
\left\|f \mid L_{q, w}(G)\right\| \leqslant C\left[\sum_{|\alpha|=s}\left\|D^{\alpha} f\left|L_{p, v}(G)\|+\| f\right| L_{r, w}\left(G_{\delta}\right)\right\|\right]
$$

или оценки слабого типа

$$
\sup _{\lambda>0} \lambda|\{x \in G:|f(x)|>\lambda\}|_{w}^{\frac{1}{q}} \leqslant C\left[\sum_{|\alpha|=s}\left\|D^{\alpha} f\left|L_{p, v}(G)\|+\| f\right| L_{r, u}\left(G_{\delta}\right)\right\|\right]
$$

справедливых для всех функций $f$ с конечной правой частью и с постоянной $C$, не зависящей от $f$.

\section{§1. Основные результаты}

Будем пользоваться следующими обозначениями:

$$
\rho(x)=\operatorname{dist}\left(x, \mathbb{R}^{n} \backslash G\right), \quad \rho_{1}(x)=\min \{\rho(x), 1\}, \quad B(x, R)=\{y:|y-x|<R\}
$$


$\chi$ - характеристическая функция $B(0,1), 1 / p^{\prime}=1-1 / p, \varepsilon>0$,

$$
\begin{gathered}
a_{+}^{(\varepsilon)}=\left\{\begin{array}{l}
a_{+} \quad \text { при } a \neq 0, \\
\varepsilon \quad \text { при } a=0,
\end{array} \quad a_{-}^{(\varepsilon)}=\left\{\begin{array}{l}
a_{-} \text {при } a \neq 0, \\
-\varepsilon \text { при } a=0,
\end{array}\right.\right. \\
Q(x, r)=x+[-r, r]^{n}, \quad M_{w} f(x)=\sup _{R>0}|G \cap B(x, R)|_{w}^{-1} \int_{G \cap B(x, R)}|f(y)| d y .
\end{gathered}
$$

Заметим, что

$$
\left|\rho_{1}(x)-\rho_{1}(y)\right| \leqslant|x-y|
$$

и что при любом $\varepsilon \in(0,1)$

$$
(1-\varepsilon) \rho_{1}(y) \leqslant(1+\varepsilon) \rho_{1}(x) \text { для } x, y \in G, \quad B\left(x, \varepsilon \rho_{1}(x)\right) \cap B\left(y, \varepsilon \rho_{1}(y)\right) \neq \varnothing \text {. }
$$

ОПРЕДЕЛЕНИЕ 1.1. Пусть область $G \subset \mathbb{R}^{n}, \delta \in(0,1), R_{0}>0, C_{0} \geqslant 1$ и каждой точке $x \in G$ поставлены в соответствие кусочно гладкий путь $\gamma=\gamma_{x}:\left[0, t_{x}\right] \rightarrow G$ и непрерывная кусочно гладкая функция $r_{\gamma}:\left[0, t_{x}\right] \rightarrow(0, \infty)$ со свойствами:

$$
\begin{gathered}
\gamma(0)=x, \quad \gamma \subset B\left(x, R_{0}\right), \quad \rho\left(\gamma\left(t_{x}\right)\right) \geqslant \delta, \quad\left|\gamma^{\prime}\right| \leqslant 1 \text { п.в., } \\
0<r_{\gamma}(t) \leqslant \delta \rho_{1}(\gamma(t)), \quad\left|r_{\gamma}^{\prime}(t)\right|=\left|\frac{d}{d t} r_{\gamma}(t)\right| \leqslant C_{0} \text { для п.в. } t \in\left(0, t_{x}\right], r_{\gamma}\left(t_{x}\right) \geqslant \delta^{2}, \\
\delta r_{\gamma}\left(t^{\prime}\right) \leqslant r_{\gamma}\left(t^{\prime \prime}\right) \text { при } B\left(\gamma\left(t^{\prime}\right), \delta r_{\gamma}\left(t^{\prime}\right)\right) \cap B\left(\gamma\left(t^{\prime \prime}\right), \delta r_{\gamma}\left(t^{\prime \prime}\right)\right) \neq \varnothing, t^{\prime}, t^{\prime \prime} \in\left[0, t_{x}\right] .
\end{gathered}
$$

Тогда будем писать $\left(G, \gamma, r_{\gamma}\right) \in \mathscr{G}\left(\delta, R_{0}, C_{0}\right)$.

ЗАмЕчАнИЕ 1.1. В качестве $r_{\gamma}(t)$ можно взять, например, функцию, эквивалентную $\rho_{1}(\gamma(t))$. Покажем это. Обозначим через $\widetilde{\rho}(x), x \in G$, регуляризованное расстояние от $x$ до $\mathbb{R}^{n} \backslash G$ (см. [14]). Функция $\widetilde{\rho} \in C^{\infty}(G)$ и при некотором $N \geqslant 1$

$$
\frac{1}{N} \rho(x) \leqslant \widetilde{\rho}(x) \leqslant \rho(x), \quad|\operatorname{grad} \widetilde{\rho}|<N \quad(x \in G) .
$$

В случае ограниченной области $G$ можно взять $r_{\gamma}(t)=\frac{1}{2} \widetilde{\rho}(\gamma(t))$. В общем случае положим $\widetilde{\rho}_{1}(x)=\varphi\left(\widetilde{\rho}_{1}(x)\right)$, где $\varphi \in C^{\infty}(0, \infty), \varphi(t)=t$ при $0<t<\frac{1}{2}, \varphi(t)=1$ при $t \geqslant 2, \frac{1}{2}<\varphi(t) \leqslant 1$ при $\frac{1}{2}<t<2, \varphi$ не убывает на $(0, \infty)$. Ясно, что $\widetilde{\rho}_{1} \in C^{\infty}(G)$ и что при некотором $N_{1}>0$

$$
\frac{1}{N_{1}} \rho_{1}(x) \leqslant \widetilde{\rho}_{1}(x) \leqslant \rho_{1}(x), \quad\left|\operatorname{grad} \widetilde{\rho}_{1}\right| \leqslant N
$$

Учитывая (1.2), завершаем доказательство замечания 1.1.

Укажем сначала простое условие, связывающее $r, q, u, w$, которое будем считать выполненным для $\left(G, \gamma, r_{\gamma}\right) \in \mathscr{G}\left(\delta, R_{0}, C_{0}\right)$ :

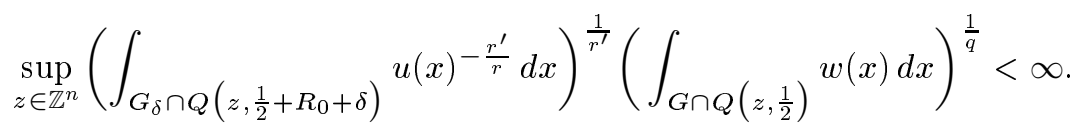

Связь между $p, q, v, w$ и геометрическими свойствами области $G$ будет иметь более сложньй вид. 
ЛЕмма 1. Пусть $(G, \gamma, r) \in \mathscr{G}\left(\delta, R_{0}, C_{0}\right)$ и выполнены условия: (2), (1.6),

$$
\begin{aligned}
& \sup _{x \in G} \sup _{R>0} \rho_{1}(x)^{(s-n)_{+}^{(\varepsilon)}}\left(\int_{B\left(x, \delta \rho_{1}(x)\right) \backslash B(x, R)}|y-x|^{(s-n)_{-}^{(\varepsilon)} p^{\prime}} v(y)^{-\frac{p^{\prime}}{p}} d y\right)^{\frac{1}{p^{\prime}}} \\
& \times|B(x, R)|_{w}^{\frac{1}{q}}<\infty, \\
& \sup _{x \in G} \sup _{R>0}\left[\int_{0}^{t_{x}}\left(t+r_{\gamma}(0)\right)^{(s-1) p^{\prime}} r_{\gamma}(t)^{\frac{1-n}{p} p^{\prime}} V_{R}\left(\gamma, r_{\gamma}(t), t\right)^{p^{\prime}} d t\right]^{\frac{1}{p^{\prime}}}|B(x, R)|_{w}^{\frac{1}{q}}<\infty,
\end{aligned}
$$

$2 \partial e$

$$
V_{R}\left(\gamma, r_{\gamma}(t), t\right)=r_{\gamma}(t)^{-\frac{n}{p^{\prime}}}\left[\int_{G \backslash B(x, R)} \chi\left(\frac{y-\gamma(t)}{r_{\gamma}(t)}\right) v(y)^{-\frac{p^{\prime}}{p}} d y\right]^{\frac{1}{p^{\prime}}}
$$

Тогда существует постоянная $C>0$ такая, что для почти всех $x \in G$

$$
\begin{aligned}
|f(x)| \leqslant C & {\left[M_{w}\left(\sum_{|\alpha|=s}\left|D^{\alpha} f\right|^{p} v\right)(x)\right]^{\frac{1}{q}}\left\|\sum_{|\alpha|=s}\left|D^{\alpha} f\right| v^{\frac{1}{p}} \mid L_{p}(G)\right\|^{1-\frac{p}{q}} } \\
& +C\left\|f \mid L_{1}\left(G_{\delta} \cap B\left(x, R_{0}+\delta\right)\right)\right\|
\end{aligned}
$$

для любой функции $f$ с конечной правой частью.

ЗАмЕчАниЕ 1.2 . При $p=1, p^{\prime}=\infty$ условие (1.8) понимается как

$$
\begin{aligned}
& \sup _{x \in G} \sup _{R>0} \sup _{0 \leqslant t \leqslant t_{x}}\left(t+r_{\gamma}(0)\right)^{s-1} r_{\gamma}(t)^{1-n} \\
& \quad \times \sup \left\{v(y)^{-1}: y \in G \backslash B(x, R),|y-\gamma(t)|<r_{\gamma}(t)\right\}|B(x, R)|_{w}^{\frac{1}{q}}<\infty .
\end{aligned}
$$

ТЕОРЕМа 1. В условиях леммы 1 существует постоянная $C>0$, при которой имеет место неравенство (4).

Теорема 2. В условиях леммы 1 при $p<\widetilde{q}<q$ или при $s=1 u \tilde{q}=q$ существует постоянная $C>0$ такая, что имеет место неравенство

$$
\left\|f\left|L_{\widetilde{q}, w}(G)\left\|\leqslant C \sum_{|\alpha|=s}\right\| D^{\alpha} f\right| L_{p, v}(G)\right\|+C\left\|f \mid L_{r, u}\left(G_{\delta}\right)\right\|
$$

для всех функиий $f$ с конечной правой частью.

При этом условие (1.1) можно ослабить, заменив в нем q на $\widetilde{q}$.

ОПРЕДЕЛЕНИЕ 1.2. При $\sigma \geqslant 1$ область $G \subset \mathbb{R}$ назовем областью с условием гибкого $\sigma$-конуса, если при некоторых $t^{*}>0, \varkappa>0$ для любого $x \in G$ сушествует кусочно гладкий путь $\gamma:\left[0, t^{*}\right] \rightarrow G, \gamma(0)=x,\left|\gamma^{\prime}\right| \leqslant 1$ почти всюду, такой, что $\rho(\gamma(t)) \geqslant \varkappa t^{\sigma}$ при $0<t \leqslant t^{*}$. 
ОПРЕДЕЛЕНИЕ 1.3 [8]. При $\sigma \geqslant 1$ область $G \subset \mathbb{R}^{n}$ называется областью $c$ $\sigma$-условием Джсона ( $\sigma$-John domain), если существуют точка $x^{(0)} \in G$ и постоянная $\varkappa>0$ такие, что для любой точки $x \in G$ найдется спрямляемая кривая $\gamma:\left[0, l_{x}\right] \rightarrow G, \gamma(0)=x, \gamma\left(l_{x}\right)=x^{(0)}$, параметризованная с помощью длины дуги и такая, что

$$
\rho(\gamma(t)) \geqslant \varkappa t^{\sigma} \text { при } 0<t<l_{x} .
$$

Заметим, что область с $\sigma$-условием Джона является также областью с условием гибкого $\sigma$-конуса.

В случае весовых функций $v(x)=\rho_{1}(x)^{a}, w(x)=\rho_{1}(x)^{b}$ справедливы следуюшие две теоремы об оценке

$$
\left\|\rho_{1}^{\frac{b}{q}} f\left|L_{q}(G)\left\|\leqslant C \sum_{|\alpha|=s}\right\| \rho_{1}^{\frac{a}{p}} D^{\alpha} f\right| L_{p}(G)\right\|+C\left\|f \mid L_{r}\left(G_{\delta}\right)\right\|
$$

для всех функций $f$ c конечной правой частью.

ТеОРема 3. Пусть для области $G$ с условием гибкого б-конуса $(\sigma \geqslant 1)$ выполнень условия (2) при $s=1$ и условия

$$
\begin{aligned}
a \in(-\infty, \infty), \quad & b \geqslant 0, \quad 1-\frac{n}{p}+\frac{n}{q} \geqslant 0, \quad 1-\frac{n+a}{p}+\frac{n+b}{q} \geqslant 0, \\
& 1-\frac{\sigma(n-1)+1+\sigma a}{p}+\frac{n+b}{q} \geqslant 0 .
\end{aligned}
$$

Тогда имеет место неравенство (1.12) при $s=1$ с некоторьми $C, \delta>0$, не зависячими от $f$.

ТЕОРема 4. Пусть для области $G$ с условием гибкого $\sigma$-конуса $(\sigma \geqslant 1)$ при $s \in \mathbb{N}, p>1$ выполнены условия (2), $a \in(-\infty, \infty), b \geqslant 0$. Пусть также выполнены условия:
i) $s-\frac{n}{p}+\frac{n}{q}>0$
ii) $s-\frac{n+a}{p}+\frac{n+b}{q}>0$;
iii) $s-\frac{\sigma(n-1+a)+1}{p}+\frac{n+b}{q} \geqslant 0$.

Тогда имеет место неравенство (1.12) при некоторых $C, \delta>0$, не зависящих от $f$.

При этом в случаях $a \geqslant 1-n$ или $\sigma=1$ условие іi) может быть снято, $а$ в случае $a=-\frac{\sigma-1}{\sigma}(n-1), b=0$ условие і) может бъть снято.

Д. А. Лабутиным [15] показано, что в невесовом случае $(a=b=0)$ показатель $q$ в теоремах 3,4 для области $G$ с $\sigma$-условием Джона нельзя увеличить.

Tеорема 5. Пусть при $1 \leqslant p, q<\infty, 1 \leqslant p_{1}<q_{1}<q<\infty, w=1$ выполнены условия леммы 1 , а также условия (1.7), (1.8), в которых $p, q$ заменены соответственно на $p_{1}, q_{1}$.

Тогда существует постоянная $C>0$, при которой имеет место неравенство (3) c $w=1$. 
Теорема 6. Пусть $(G, \gamma, r) \in \mathscr{G}\left(\delta, R_{0}, C_{0}\right)$ и выполнены условия (2), (1.6) и

$$
\sum_{j=j_{0}}^{\infty}\left(\xi_{j}+\eta_{j}\right)<\infty
$$

әде $j_{0} \in \mathbb{Z}$ определено соотношением $2^{-j_{0}-1}<R_{0}+\delta \leqslant 2^{-j_{0}}$,

$$
\begin{aligned}
& \xi_{j}=\sup _{x \in G} \rho_{1}(x)^{(s-n)_{+}^{(\varepsilon)}}\left(\int_{B\left(x, \delta \rho_{1}(x)\right) \backslash B\left(x, 2^{-j}\right)}|y-x|^{(s-n)_{-}^{(\varepsilon)} p^{\prime}} v(y)^{-\frac{p^{\prime}}{p}} d y\right)^{\frac{1}{p^{\prime}}} \\
& \quad \times\left|B\left(x, 2^{-j+1}\right)\right|_{w}^{\frac{1}{q}} \quad(\varepsilon>0), \\
& \eta_{j}=\sup _{x \in G}\left(\int_{0}^{t_{x}}\left(t+r_{\gamma}(0)\right)^{(s-1) p^{\prime}} r_{\gamma}(t)^{\frac{1-n}{p} p^{\prime}} V_{2^{-j}}\left(\gamma, r_{\gamma}(t), t\right)^{p^{\prime}} d t\right)^{\frac{1}{p^{\prime}}}\left|B\left(x, 2^{-j+1}\right)\right|_{w}^{\frac{1}{q}} .
\end{aligned}
$$

Тогда существует постоянная $C>0$, при которой справедливо неравенство (3).

\section{§ 2. Интегральные представления функций и поточечные оценки}

Построим сначала интегральное представление функции через первые производные по гибкому конусу, обобщаюшее соответствующее интегральное представление функции через первые производные из [5; п. 7.3]. Пусть $\omega \in C_{0}^{\infty}(\mathbb{R}), \operatorname{supp} \omega \subset$ $\left[-\frac{1}{\sqrt{n}}, \frac{1}{\sqrt{n}}\right], \int \omega(u) d u=1, \widehat{\omega}(u)=u \omega^{\prime}(u)$, функции $\tau:\left[0, t^{*}\right] \rightarrow \mathbb{R}, r:\left[0, t^{*}\right] \rightarrow$ $[0, \infty)$ непрерывны, $r(0)=0, r(t)>0$ при $t>0$. В точке $t$ дифференцируемости функций $\tau, r$ имеем

$$
\begin{aligned}
& \frac{\partial}{\partial t}\left[\frac{1}{r(t)} \omega\left(\frac{v-\tau(t)}{r(t)}\right)\right]=-\frac{r^{\prime}(t)}{r(t)^{2}}\left[\omega\left(\frac{v-\tau(t)}{r(t)}\right)+\frac{v-\tau(t)}{r(t)} \omega^{\prime}\left(\frac{v-\tau(t)}{r(t)}\right)\right] \\
& \quad-\frac{\tau^{\prime}(t)}{r(t)^{2}} \omega^{\prime}\left(\frac{v-\tau(t)}{r(t)}\right)=-\frac{r^{\prime}(t)}{r(t)^{2}} \widehat{\omega}^{\prime}\left(\frac{v-\tau(t)}{r(t)}\right)-\frac{\tau^{\prime}(t)}{r(t)^{2}} \omega^{\prime}\left(\frac{v-\tau(t)}{r(t)}\right) \\
& =-\frac{\partial}{\partial v}\left[\frac{r^{\prime}(t)}{r(t)} \widehat{\omega}\left(\frac{v-\tau(t)}{r(t)}\right)+\frac{\tau^{\prime}(t)}{r(t)} \omega\left(\frac{v-\tau(t)}{r(t)}\right)\right] \\
& =-\frac{\partial}{\partial v} k\left(\frac{v-\tau(t)}{r(t)}, \frac{r^{\prime}(t)}{r(t)}, \frac{\tau^{\prime}(t)}{r(t)}\right) .
\end{aligned}
$$

При этом

$$
|k(\eta, a, b)| \leqslant C(|a|+|b|)\left(|\omega(\eta)|+\left|\omega^{\prime}(\eta)\right|\right) .
$$

Пусть теперь $x \in G, \gamma:\left[0, t_{x}\right] \rightarrow G$ - кусочно гладкий путь со свойствами (1.2), $r:[0, t] \rightarrow[0, \infty)-$ кусочно гладкая функция,

$$
\begin{gathered}
r(0)=0, \quad 0<r(t) \leqslant \frac{1}{2} \rho(\gamma(t)), \quad\left|r^{\prime}(t)\right| \leqslant C_{0} \quad \text { при } t \in\left(0, t_{x}\right] \\
f_{t}(x)=r(t)^{-n} \int \prod_{j=1}^{n} \omega\left(\frac{y_{j}-\left(\gamma_{j}(t)-x_{j}\right)}{r(t)}\right) f(x+y) d y \\
=r(t)^{-n} \int \Omega\left(\frac{y-\gamma(t)}{r(t)}\right) f(y) d y
\end{gathered}
$$


Представим функцию $f$ в точке $x$ в виде

$$
f(x)=-\int_{0}^{t_{x}} \frac{\partial}{\partial t} f_{t}(x) d t+f_{t_{x}}(x)
$$

В силу (2.1) и интегрирования по частям имеем при $0<t \leqslant t_{x}$

$$
\begin{aligned}
& \frac{\partial}{\partial t} f_{t}(x)=\sum_{i=1}^{n} \int \frac{\partial}{\partial y_{i}} k\left(\frac{y_{i}-\gamma_{i}(t)}{r(t)}, \frac{r^{\prime}(t)}{r(t)}, \frac{\gamma_{i}^{\prime}(t)}{r(t)}\right) \prod_{j \neq i} \frac{1}{r(t)} \omega\left(\frac{y_{j}-\gamma_{j}(t)}{r(t)}\right) f(y) d y \\
& =-\sum_{i=1}^{n} r(t)^{1-n} \int k\left(\frac{y_{i}-\gamma_{i}(t)}{r(t)}, \frac{r^{\prime}(t)}{r(t)}, \frac{\gamma_{i}^{\prime}(t)}{r(t)}\right) \prod_{j \neq i} \omega\left(\frac{y_{j}-\gamma_{j}(t)}{r(t)}\right) D_{i} f(y) d y .
\end{aligned}
$$

Отсюда в силу (2.2) и ограниченности $\left|r^{\prime}\right|,\left|\gamma^{\prime}\right|$ получаем оценку

$$
\left|\frac{\partial}{\partial t} f_{t}(x)\right| \leqslant C r(t)^{-n} \int \chi\left(\frac{y-\gamma(t)}{r(t)}\right) \sum_{i=1}^{n}\left|D_{i} f(y)\right| d y
$$

Из (2.5), (2.7) следует, что

$$
|f(x)| \leqslant C \int_{0}^{t_{x}} r(t)^{-n} \int_{|y-\gamma(t)|<r(t)} \sum_{i=1}^{n}\left|D_{i} f(y)\right| d y d t+\left|f_{t_{x}}(x)\right| .
$$

Лемма 2.1. Пусть $s \in \mathbb{N}, x \in G, \gamma:\left[0, t_{x}\right] \rightarrow G-$ кусочно гладкий путь со свойствами (1.2), $r:\left[0, t_{x}\right] \rightarrow \mathbb{R}-$ кусочно гладкая функиия со свойствами (2.3). Тогда

$$
\begin{aligned}
|f(x)| \leqslant C & \int_{0}^{t_{x}} t^{s-1} r(t)^{-n} \int_{|y-\gamma(t)|<r(t)} \sum_{|\alpha|=s}\left|D^{\alpha} f(y)\right| d y d t \\
& +C \int_{\left|y-\gamma\left(t_{x}\right)\right|<r\left(t_{x}\right)}|f(y)| d y,
\end{aligned}
$$

әде $C$ не зависит от $f, x$.

ДокАЗАТЕЛЬСтво. Установим сначала, что в условиях леммы

$$
\begin{aligned}
|f(x)| \leqslant & C \int_{0}^{t_{x}} t^{s-1} r(t)^{-n} \int_{|y-\gamma(t)|<r(t)} \sum_{|\alpha|=s}\left|D^{\alpha} f(y)\right| d y d t \\
& +C \sum_{|\alpha| \leqslant s-1}\left|\left(D^{\alpha} f\right)_{t_{x}}(x)\right|,
\end{aligned}
$$

где $C$ не зависит от $f, x$. При $s=1$ оценка (2.10) совпадает с (2.8). Предположим, что $s \geqslant 2$ и что оценка (2.10) верна с $s-1$ вместо $s$. Зафиксируем в ее подынтегральном выражении $t, y(|y-\gamma(t)|<r(t))$. Воспользуемся для оценки $D^{\beta} f(y)$ с 
$|\beta|=s-1$ оценкой $(2.8)$, в которой в качестве пути возьмем $\gamma_{t}:\left[0, u^{*}\right] \rightarrow G$, где $u^{*}=r(t)+t_{x}-t$,

$$
\gamma_{t}(u)= \begin{cases}y+(\gamma(t)-y) \frac{u}{u_{*}}=y+\frac{\gamma(t)-y}{|\gamma(t)-y|} u & \text { при } 0 \leqslant u \leqslant u_{*}=|y-\gamma(t)|, \\ \gamma(t) & \text { при } u_{*} \leqslant u \leqslant r(t), \\ \gamma(t+u-r(t)) & \text { при } r(t) \leqslant u \leqslant u^{*} .\end{cases}
$$

Построим непрерывную кусочно гладкую функцию $r_{t}:\left[0, u^{*}\right] \rightarrow \mathbb{R}$, положив

$$
r_{t}(u)= \begin{cases}u & \text { при } 0<u \leqslant r(t), \\ r(t+u-r(t)) & \text { при } r(t) \leqslant u \leqslant u^{*} .\end{cases}
$$

Тогда $\left|\frac{d}{d u} r_{t}(u)\right| \leqslant C_{0}, r_{t}(u) \leqslant C_{0} u, r_{t}\left(u^{*}\right) \geqslant \delta$.

Применяя оценку (2.8), имеем

$$
\left|D^{\beta} f(y)\right| \leqslant C_{1} \int_{0}^{u^{*}} r_{t}(u)^{-n} \int_{\left|z-\gamma_{t}(u)\right| \leqslant r_{t}(u)} \sum_{|\alpha|=s}\left|D^{\alpha} f(z)\right| d z d u+\left|\left(D^{\beta} f\right)_{u^{*}}(y)\right| .
$$

Подставляя $r_{t}, \gamma_{t}(u)$ из их определений, имеем отсюда

$$
\begin{aligned}
\left|D^{\beta} f(y)\right| \leqslant & C_{2}\left(\int_{0}^{r(t)} u^{-n}+\int_{r(t)}^{u^{*}} r_{t}(u)^{-n}\right) \\
& \times \int_{\left|z-\gamma_{t}(u)\right| \leqslant r_{t}(u)} \sum_{|\alpha|=s}\left|D^{\alpha} f(z)\right| d z d u+\left|\left(D^{\beta} f\right)_{t_{x}}(x)\right| \\
\leqslant & C_{2} \int_{|z-\gamma(t)|<r(t)} \int_{\frac{1}{2}|z-y|}^{r(t)} u^{-n} \sum_{|\alpha|=s}\left|D^{\alpha} f(z)\right| d u d z \\
& +C_{2} \int_{\left[r(t), r(t)+t_{x}-t\right]} r(t+u-r(t))^{-n} \\
& \times \int_{|z-\gamma(t+u-r(t))|<r(t+u-r(t))} \sum_{|\alpha|=s}\left|D^{\alpha} f(z)\right| d z d u+\left|\left(D^{\beta} f\right)_{t_{x}}(x)\right| .
\end{aligned}
$$

После сдвигов по $u$ имеем

$$
\begin{aligned}
\left|D^{\beta} f(y)\right| \leqslant & C_{3} \int_{|z-\gamma(t)|<r(t)}|z-y|^{1-n} \sum_{|\alpha|=s}\left|D^{\alpha} f(z)\right| d z \\
& +C_{2} \int_{t}^{t_{x}} r(v)^{-n} \int_{|z-\gamma(v)|<r(v)} \sum_{|\alpha|=s}\left|D^{\alpha} f(z)\right| d z d v+\left|\left(D^{\beta} f\right)_{t_{x}}(x)\right| .
\end{aligned}
$$

Отсюда

$$
\begin{aligned}
& \int_{|y-\gamma(t)|<r(t)}\left|D^{\beta} f(y)\right| d y \leqslant C_{4} r(t) \int_{|z-\gamma(t)|<r(t)} \sum_{|\alpha|=s}\left|D^{\alpha} f(z)\right| d z \\
& +C_{4} r(t)^{n} \int_{t}^{t_{x}} r(u)^{-n} \int_{|z-\gamma(u)|<r(u)} \sum_{|\alpha|=s}\left|D^{\alpha} f(z)\right| d z d u \\
& +C_{4} r(t)^{n}\left|\left(D^{\beta} f\right)_{t_{x}}(x)\right| .
\end{aligned}
$$


Подставляя последнюю оценку в неравенство (2.10), написанное для $s-1$ вместо $s$, получаем

$$
\begin{aligned}
|f(x)| \leqslant & C \int_{0}^{t_{x}} t^{s-2} r(t)^{1-n} \int_{|z-\gamma(t)|<r(t)} \sum_{|\alpha|=s}\left|D^{\alpha} f(z)\right| d z d t \\
& +C \int_{0}^{t_{x}} t^{s-2} \int_{t}^{t_{x}} r(u)^{-n} \int_{|z-\gamma(u)|<r(u)} \sum_{|\alpha|=s}\left|D^{\alpha} f(z)\right| d z d u d t \\
& +C \sum_{|\alpha| \leqslant s-1}\left|\left(D^{\alpha} f\right)_{t_{x}}(x)\right| .
\end{aligned}
$$

Меняя порядок интегрирования во втором слагаемом и вычисляя интеграл по $t$, получаем оценку (2.10).

Оценим слагаемые $\left(D^{\alpha} f\right)_{t_{x}}(x),|\alpha| \leqslant s-1$, из правой части (2.10). Используя определение (2.4) и применяя при $0<|\alpha| \leqslant s-1$ интегрирование по частям, получаем, что

$$
\left|\left(D^{\alpha} f\right)_{t_{x}}(x)\right| \leqslant C_{\alpha} \int_{G} \chi\left(\frac{y-\gamma\left(t_{x}\right)}{r\left(t_{x}\right)}\right)|f(y)| d y, \quad|\alpha| \leqslant s-1 .
$$

Отсюда и из (2.10) получаем оценку (2.9), чем и завершается доказательство леммы 2.1.

Лемма 2.2. Пусть $s \in \mathbb{N}, x \in G, \gamma:\left[0, t_{x}\right] \rightarrow G-$-уссочно гладкий путь со свойствами $(1.2), r_{\gamma}:\left[0, t_{x}\right] \rightarrow(0, \infty)$ - кусочно гладкая функция со свойствами (1.4). Тогда

$$
|f(x)| \leqslant C A_{1}\left(\sum_{|\alpha|=s}\left|D^{\alpha} f\right|\right)(x)+C A_{2}\left(\sum_{|\alpha|=s}\left|D^{\alpha} f\right|\right)(x)+C A_{3} f(x),
$$

2дe

$$
\begin{aligned}
& A_{1} g(x)=\int_{0}^{r_{\gamma}(0)} t^{s-1-n} \int_{|y-x|<t} g(y) d y d t, \\
& A_{2} g(x)=\int_{0}^{t_{x}}\left(t+r_{\gamma}(0)\right)^{s-1} r_{\gamma}(t)^{-n} \int_{|y-\gamma(t)|<r_{\gamma}(t)} g(y) d y d t \\
& A_{3} f(x)=\int_{\left|y-\gamma\left(t_{x}\right)\right|<r_{\gamma}\left(t_{x}\right)}|f(y)| d y .
\end{aligned}
$$

ДокАЗАТЕЛЬСТво. Пусть $x \in G$. По данному пути $\gamma$ со свойствами (1.2) построим путь

$$
\gamma_{0}(t)= \begin{cases}\gamma(0) & \text { при } 0 \leqslant t \leqslant r_{\gamma}(0), \\ \gamma\left(t-r_{\gamma}(0)\right) & \text { при } r_{\gamma}(0) \leqslant t \leqslant t_{x}+r_{\gamma}(0) .\end{cases}
$$

Свяжем с путем $\gamma_{0}$ кусочно гладкую функцию

$$
r(t)= \begin{cases}t & \text { при } 0 \leqslant t \leqslant r_{\gamma}(0), \\ r_{\gamma}\left(t-r_{\gamma}(0)\right) & \text { при } r_{\gamma}(0) \leqslant t<t_{x}+r_{\gamma}(0) .\end{cases}
$$

Подставив $\gamma_{0}$ (вместо $\left.\gamma\right)$ и $r(t)$ в $(2.9)$, получим $(2.11)$. 
ЗАмечАниЕ 2.1. Разбив отрезок $\left[0, t_{x}\right]$ интегрирования в $(2.13)$ на два точкой $l_{x}=\min \left\{t_{x}, \frac{1}{2 C_{0}} r_{\gamma}(0)\right\}$, где $C_{0}>1$ то же, что в $(1.3)$, легко получим оценку

$$
A_{2} g(x) \leqslant C_{1} A_{2,1} g(x)+C_{1} A_{2,2} g(x) \quad(g \geqslant 0)
$$

где

$$
\begin{aligned}
& A_{2,1} g(x)=r_{\gamma}(0)^{s-1-n} \int_{|y-x|<2 r_{\gamma}(0)} g(y) d y \\
& A_{2,2} g(x)=\int_{l_{x}}^{t_{x}} t^{s-1} r_{\gamma}(t)^{-n} \int_{|y-\gamma(t)|<r_{\gamma}(t)} g(y) d y d t
\end{aligned}
$$

Тогда оценку (2.11) можно заменить на аналогичную оценку $|f(x)|$ с помощью операторов $A_{1}, A_{2,1}, A_{2,2}, A_{3}$. При последующих оценках этих операторов лишь оценка $A_{2,2}$ требует учета специфики области $G$.

\section{§ 3. Исправление гибкого конуса представления}

Можно сказать, что оценка (2.11) основана на представлении функции $f(x)$ по "усеченному гибкому конусу"

$$
\left(\gamma, r_{\gamma}\right):=\bigcup_{0 \leqslant t \leqslant t_{x}} B\left(\gamma(t), r_{\gamma}(t)\right) \subset G
$$

построенному по пути $\gamma$. Для дальнейших оценок $|f(x)|$ в качестве усеченного гибкого конуса представления будет избран некоторый правильный усеченный гибкий конус $\left(\widehat{\gamma}, r_{\widehat{\gamma}}\right)$, полученньй исправлением $\left(\gamma, r_{\gamma}\right)$. Способ исправления по существу совпадает с описанньм в [10] способом исправления червя (a worm). Путь $\widehat{\gamma}$ будет представлять собой вписанную в $\gamma$ ломаную, построенную следующим образом.

Зафиксируем $\varepsilon \in\left(0, \frac{1}{2}\right]$. Возьмем в качестве последовательных вершин $\widehat{\gamma}$ точки $\gamma\left(t_{i}\right)$,

$$
0=t_{0}<t_{1}<\cdots<t_{m}=t_{x}
$$

где значения $t_{i}$ выберем следуюшим образом. Положим $t_{0}=0$. Пусть $t_{1}, \ldots, t_{i-1}$ уже выбраны. Если $t_{i-1}=t_{x}$, то положим $m=i-1, t_{m}=t_{x}$ и построение закончено. Если $t_{i-1}<t_{x}$, то положим

$$
t_{i}=\sup \left\{t \in\left(t_{i-1}, t_{x}\right]: B\left(\gamma(t), \varepsilon r_{\gamma}(t)\right) \cap B\left(\gamma\left(t_{i-1}\right), \varepsilon r_{\gamma}\left(t_{i-1}\right)\right) \neq \varnothing\right\}
$$

Легко видеть, что число шагов $m$ конечно. Можно сказать, что построена "нить бус" из $B_{i}$, где $B_{i}=B\left(\gamma\left(t_{i}\right), \varepsilon r_{\gamma}\left(t_{i}\right)\right)(i=1,2, \ldots, m)$. При этом выполняются свойства:

(i) $B_{i} \cap B_{j}=\varnothing$ при $0 \leqslant i, j \leqslant m-1, i \neq j$,

(ii) $\bar{B}_{i-1} \cap \bar{B}_{i} \neq \varnothing(i=1,2, \ldots, m)$,

(iii) $\delta r_{\gamma}\left(t_{i}\right) \leqslant r_{\gamma}\left(t_{j}\right)$ при $\left|\gamma\left(t_{i}\right)-\gamma\left(t_{j}\right)\right| \leqslant 2 \varepsilon r_{\gamma}\left(t_{i}\right)+2 \varepsilon r_{\gamma}\left(t_{j}\right)$,

(iv) $B\left(\gamma\left(t_{i}\right), r_{\gamma}\left(t_{i}\right)\right) \subset G$ при $i=0,1, \ldots, m$. 
Через $\widehat{\gamma}:\left[0, \tau_{x}\right] \rightarrow G$ обозначим путь, состоящий из отрезков, последовательно соединяющих центры шаров $B_{i}$, и параметризованный с помощью переменной длины дуги, отсчитываемой от $x=\gamma(0)=\widehat{\gamma}(0)$. Пусть при этом $\left\{\tau_{i}\right\}_{0}^{m}-$ значения параметров последовательных вершин $\widehat{\gamma}$, так что

$$
\widehat{\gamma}\left(\tau_{i}\right)=\gamma\left(t_{i}\right), \quad 0=\tau_{0}<\tau_{1}<\cdots<\tau_{m}=\tau_{x}
$$

Через $r_{\widehat{\gamma}}:\left[0, \tau_{x}\right] \rightarrow(0, \infty)$ обозначим непрерывную функцию, принимающую значения $r_{\widehat{\gamma}}\left(\tau_{i}\right)=\varepsilon r_{\gamma}\left(t_{i}\right)(i=0,1, \ldots, m)$ и линейную на каждом отрезке $\left[\tau_{i-1}, \tau_{i}\right]$.

Построенный усеченный гибкий конус $\left(\widehat{\gamma}, r_{\widehat{\gamma}}\right)$ будем называть $\varepsilon$-исправлением $\left(\gamma, r_{\gamma}\right)$, а также $\varepsilon$-исправленным. Он является $\varepsilon$-правильньм в смысле следуюшего определения.

ОПРЕДЕЛЕНИЕ 3.1. Пусть $\varepsilon \in\left(0, \frac{1}{2}\right]$. Усеченный гибкий конус $\left(\gamma, r_{\gamma}\right)$ назовем $\varepsilon$-правильнылм, если $\gamma:\left[0, t_{x}\right] \rightarrow G$ - ломаная со свойствами $(1.3), r_{\gamma}:\left[0, t_{x}\right] \rightarrow$ $(0, \infty)$ - кусочно гладкая функция со свойствами $(1.4),(1.5)$ и при некотором разбиении $0=t_{0}<t_{1}<\cdots<t_{m}=t_{x}$ отрезка $\left.\left[0, t_{x}\right] \gamma\right|_{\left[t_{i-1}, t_{i}\right]}$ - отрезки для всех $i=1,2, \ldots, m$, а шары $B_{i}=B\left(\gamma\left(t_{i}\right), r_{\gamma}\left(t_{i}\right)\right)$ обладают свойствами (i)-(iv).

Пусть

$$
J(y)=\left\{j: j \in\{0,1, \ldots, m\}, y \in B\left(\gamma\left(t_{j}\right), 2 r_{\gamma}\left(t_{j}\right)\right)\right\}, \quad y \in G
$$

В силу свойств (i), (iii) $B_{j} \cap B_{k}=\varnothing$ при $j \neq k, r_{\gamma}\left(t_{j}\right) \geqslant \delta r_{\gamma}\left(t_{i}\right)$ при $j \in J(y)$. Отсюда следует, что сушествует число $N=N(\delta) \in \mathbb{N}$ такое, что

$$
\operatorname{card} J(y) \leqslant N(\delta) \text { при } y \in G .
$$

Последнее соотношение показывает, что "кратность самопересечения" правильного усеченного гибкого конуса ограничена числом, не зависящим от конуса.

Лемма 3.1. Пусть $\left(G, \gamma, r_{\gamma}\right) \in \mathscr{G}\left(\delta, R_{0}, C_{0}\right)$ и выполнено условие (1.8). Пусть $0<\varepsilon \leqslant \frac{\delta^{2}}{3+2 \delta},\left(\widehat{\gamma}, r_{\widehat{\gamma}}\right)-\varepsilon$-исправление $\left(\gamma, r_{\gamma}\right)$.

Тогда существует постоянная $\widehat{C}_{0}=\widehat{C}_{0}(\varepsilon)>0$ такая, что $\left(G, \widehat{\gamma}, r_{\widehat{\gamma}}\right) \in$ $\mathscr{G}\left(\varepsilon \delta, R_{0}, \widehat{C}_{0}\right)$ и условие (1.8) выполняется после заменьи в нем $\gamma$ на $\widehat{\gamma}, r_{\gamma}$ на $r_{\widehat{\gamma}}, C_{0}$ на $\widehat{C}_{0}$.

ДокАЗАТЕЛЬство. Оценим левую часть (1.8) для $\widehat{\gamma}$ через левую часть (1.8) для $\gamma$. Достаточно, очевидно, ограничиться сравнением левых частей (1.8) для сужений $\left.\gamma\right|_{\left[t_{i-1}, t_{i}\right]}$ и $\left.\widehat{\gamma}\right|_{\left[\tau_{i-1}, \tau_{i}\right]}$. Разберем лишь случай $i \leqslant m-1$, так как случай $i=m$ рассматривается аналогично. Очевидно,

$$
\tau_{i}-\tau_{i-1} \leqslant t_{i}-t_{i-1}
$$


Пусть $\tau_{i-1} \leqslant \tau \leqslant \tau_{i}, t_{i-1} \leqslant t \leqslant t_{i-1}+\tau_{i}-\tau_{i-1}$. Тогда в силу $(1.5) r_{\widehat{\gamma}}(\tau) \leqslant$ $\frac{1}{\delta} r_{\widehat{\gamma}}\left(\tau_{i-1}\right)$

$$
\begin{aligned}
& \tau_{i}-\tau_{i-1}=r_{\widehat{\gamma}}\left(\tau_{i-1}\right)+r_{\widehat{\gamma}}\left(\tau_{i}\right)=\varepsilon r_{\gamma}\left(t_{i-1}\right)+\varepsilon r_{\gamma}\left(t_{i}\right) \leqslant \varepsilon\left(1+\frac{1}{\delta}\right) r_{\gamma}\left(t_{i-1}\right) \\
& =\left(1+\frac{1}{\delta}\right) r_{\widehat{\gamma}}\left(\tau_{i-1}\right) \leqslant r_{\gamma}\left(t_{i-1}\right) \\
& V_{R}\left(\widehat{\gamma}, r_{\widehat{\gamma}}(\tau), \tau\right) \leqslant C_{1} V_{R}\left(\widehat{\gamma}, r_{\widehat{\gamma}}(\tau)+\tau-\tau_{i-1}, \tau_{i-1}\right) \\
& \leqslant C_{2} V_{R}\left(\widehat{\gamma},\left(1+\frac{2}{\delta}\right) r_{\widehat{\gamma}}\left(\tau_{i-1}\right), \tau_{i-1}\right)=C_{2} V_{R}\left(\gamma,\left(1+\frac{2}{\delta}\right) \varepsilon r_{\gamma}\left(t_{i-1}\right), t_{i-1}\right) \\
& \leqslant C_{3} V_{R}\left(\gamma,\left(1+\frac{2}{\delta}\right) \varepsilon r_{\gamma}\left(t_{i-1}\right)+t-t_{i-1}, t\right) \\
& \leqslant C_{4} V_{R}\left(\gamma,\left(2+\frac{3}{\delta}\right) \varepsilon r_{\gamma}\left(t_{i-1}\right), t\right) \leqslant C_{5} V_{R}\left(\gamma,\left(2+\frac{3}{\delta}\right) \varepsilon r_{\gamma}(t), t\right) \\
& \leqslant C_{6} \varepsilon^{-\frac{n}{p^{\prime}}} V_{R}\left(\gamma, r_{\gamma}(t), t\right) .
\end{aligned}
$$

Отсюда при $p>1\left(p^{\prime}<\infty\right)$ имеем

$$
\begin{aligned}
\left\{\int_{\tau_{i-1}}^{\tau_{i}}\left(\tau+r_{\widehat{\gamma}}(0)\right)^{(s-1) p^{\prime}} r_{\widehat{\gamma}}(\tau)^{\frac{1-n}{p} p^{\prime}} V_{R}\left(\widehat{\gamma}, r_{\widehat{\gamma}}(\tau), \tau\right)^{p^{\prime}} d \tau\right\}^{\frac{1}{p^{\prime}}} \\
\leqslant\left\{\int_{\tau_{i-1}}^{\tau_{i}}\left(\tau+\varepsilon r_{\gamma}(0)\right)^{(s-1) p^{\prime}} d \tau\right\}^{\frac{1}{p^{\prime}}} \\
\times \min \left\{r_{\gamma}(t)^{\frac{1-n}{p}} V_{R}\left(\gamma, r_{\gamma}(t), t\right): t_{i-1} \leqslant t \leqslant t_{i-1}+\tau_{i}-\tau_{i-1}\right\} \\
\leqslant C_{\varepsilon}\left\{\int_{t_{i-1}}^{t_{i}}\left(t+r_{\gamma}(0)\right)^{(s-1) p^{\prime}} r_{\gamma}(t)^{\frac{1-n}{p} p^{\prime}} V_{R}\left(\gamma, r_{\gamma}(t), t\right)^{p^{\prime}} d t\right\}^{\frac{1}{p^{\prime}}} .
\end{aligned}
$$

Учитывая (3.4), получаем утверждение леммы при $p>1$. При $p=1$ рассуждения аналогичны.

Лемма 3.2. Пусть $\left(G, \gamma, r_{\gamma}\right) \in \mathscr{G}\left(\delta, R_{0}, C_{0}\right), \gamma:\left[0, t_{x}\right] \rightarrow G,(\gamma, r)-$ правильный усеченный гибкий конус.

Тогда существуют числа $\widetilde{C} \geqslant 1, \varepsilon_{0} \in(0,1]$ такие, что

$$
I(y)=\int_{0}^{t_{x}} \frac{1}{r_{\gamma}(t)} \chi\left(\frac{y-\gamma(t)}{\varepsilon_{0} r_{\gamma}(t)}\right) d t \leqslant \widetilde{C},
$$

әде $\widetilde{C}, \varepsilon_{0}$ не зависят от $\gamma$ и $у$.

ДоКАЗАТЕЛЬСтво. В силу (3.3)

$$
\begin{aligned}
I(y) & \leqslant \sum_{j \in J(y)} \frac{1}{\min \left\{r_{\gamma}\left(t_{j-1}\right), r_{\gamma}\left(t_{j}\right)\right\}} \int_{t_{j-1}}^{t_{j}} d t \\
& \leqslant \sum_{j \in J(y)} \frac{\max \left\{r_{\gamma}\left(t_{j-1}\right), r_{\gamma}\left(t_{j}\right)\right\}}{\min \left\{r_{\gamma}\left(t_{j-1}\right), r_{\gamma}\left(t_{j}\right)\right\}} \leqslant N \frac{2}{\delta} \leqslant \widetilde{C},
\end{aligned}
$$

что и требовалось доказать. 
$\S 4$. Оценки для операторов $A_{1}, A_{2}, A_{3}$

Рассмотрим операторы $A_{1}, A_{2}, A_{3}$ из (2.12)-(2.14).

ОПРЕДЕЛЕНИЕ 4.1. Будем писать $\left(G, \gamma, r_{\gamma}\right) \in \mathscr{G}_{0}\left(\delta, R_{0}, C_{0}\right)$, если $\left(G, \gamma, r_{\gamma}\right) \in$ $\mathscr{G}\left(\delta, R_{0}, C_{0}\right)$ (см. определение 1.1$)$ и при этом все усеченные гибкие конусы $\left(\gamma, r_{\gamma}\right)$ являются $\delta$-правильными.

В $\S 4$ будем считать, что $\left(G, \gamma, r_{\gamma}\right) \in \mathscr{G}_{0}\left(\delta, R_{0}, C_{0}\right)$. Этим обеспечивается получение желаемой оценки для оператора $A_{2}$.

Оценим сначала $A_{3} f(x)$, считая $0<\delta \leqslant \frac{1}{2}$. Учитывая (1.1), при $\left|y-\gamma\left(t_{x}\right)\right|<$ $r_{\gamma}\left(t_{x}\right) \leqslant \delta \rho_{1}\left(\gamma\left(t_{x}\right)\right)$ имеем

$$
\begin{gathered}
\left|\rho_{1}(y)-\rho_{1}\left(\gamma\left(t_{x}\right)\right)\right|<\delta \rho_{1}\left(\gamma\left(t_{x}\right)\right), \quad \rho_{1}(y)>(1-\delta) \rho_{1}\left(\gamma\left(t_{x}\right)\right)>(1-\delta) \delta \geqslant \frac{\delta}{2}, \\
|y-x| \leqslant\left|y-\gamma\left(t_{x}\right)\right|+\left|\gamma\left(t_{x}\right)-\gamma(0)\right| \leqslant \delta \rho_{1}\left(\gamma\left(t_{x}\right)\right)+R_{0} \leqslant \delta+R_{0} .
\end{gathered}
$$

Поэтому

$$
A_{3} f(x) \leqslant \bar{A}_{3} f(x)=\int_{G_{\delta}} \chi\left(\frac{y-x}{R_{0}+\delta}\right)|f(y)| d y .
$$

ЛЕмма 4.1. Пусть выполнено условие (1.6).

Тогда при некотором $C>0$

$\left\|A_{3} f\left|L_{q, w}(G)\|\leqslant\| \bar{A}_{3} f\right| L_{q, w}(G)\right\| \leqslant C\left\|f \mid L_{r, u}(G)\right\| \quad \forall f \in L_{r, u}\left(G_{\delta}\right)$.

ДокАЗАТЕЛЬСтво. Применяя неравенство Гёльдера и пользуясь условием (1.1), получаем

$$
\begin{aligned}
& \int_{G \cap Q\left(z, \frac{1}{2}\right)}\left(\bar{A}_{3} f(x)\right)^{q} w(x) d x \\
& \leqslant \sup _{x \in G \cap Q\left(z, \frac{1}{2}\right)}\left(\int_{G_{\delta}} \chi\left(\frac{y-x}{R_{0}+\delta}\right)|f(y)| d y\right)^{q} \int_{G \cap Q\left(z, \frac{1}{2}\right)} w(x) d x \\
& \leqslant\left(\int_{G_{\delta} \cap Q\left(z, \frac{1}{2}+R_{0}+\delta\right)}|f(y)|^{r} u(y) d y\right)^{\frac{q}{r}} \\
& \quad \times\left(\int_{G_{\delta} \cap Q\left(z, \frac{1}{2}+R_{0}+\delta\right)} u(y)^{-\frac{r^{\prime}}{r}} d y\right)^{\frac{q}{r^{\prime}}} \int_{G \cap Q\left(z, \frac{1}{2}\right)} w(x) d x .
\end{aligned}
$$

Отсюда, учитьвая конечность кратности покрытия $\left\{Q\left(z, \frac{1}{2}+R_{0}+\delta\right)\right\}$, имеем

$$
\begin{aligned}
& \left(\int_{G}\left(A_{3} f(x)\right)^{q} w(x) d x\right)^{\frac{1}{q}}=\left(\sum_{z \in \mathbb{Z}^{n}} \int_{G \cap Q\left(z, \frac{1}{2}\right)}\left(A_{3} f\right)^{q} w d x\right)^{\frac{1}{q}} \\
& \quad \leqslant\left(\sum_{z \in \mathbb{Z}^{n}}\left(\int_{G \cap Q\left(z, \frac{1}{2}\right)}\left(A_{3} f\right)^{q} w d x\right)^{\frac{r}{q}}\right)^{\frac{1}{r}} \\
& \quad \leqslant C_{1}\left\{\sum_{z \in \mathbb{Z}^{n}} \int_{G_{\delta} \cap Q\left(z, \frac{1}{2}+R_{0}+\delta\right)}|f|^{r} u d y\right\}^{\frac{1}{r}} \leqslant C\left(\int_{G_{\delta}}|f|^{r} u d y\right)^{\frac{1}{r}} .
\end{aligned}
$$

Лемма доказана. 
Будем говорить, что оператор $A$ имеет сильный тип $(p, v ; q, w)$, где $1 \leqslant p \leqslant$ $q<\infty, v, w: G \rightarrow(0, \infty)$ - весовые функции, если

$$
\left\|A f\left|L_{q, w}(G)\|\leqslant C\| f\right| L_{p, v}(G)\right\| \quad \forall f \in L_{p, v}(G),
$$

и что $A$ имеет слабъй тип $(p, v ; q, w)$, если

$$
\sup _{\lambda>0} \lambda|\{x \in G:|A f(x)|>\lambda\}|_{w}^{\frac{1}{q}} \leqslant C\left\|f \mid L_{p, v}(G)\right\| \quad \forall f \in L_{p, v}(G) .
$$

Последние две оценки будем назьвать соответственно оценками сильного и слабого типа $(p, v ; q, w)$. В дальнейшем мы используем интерполящионную теорему Марцинкевича, позволяющую получать оценки сильного типа с помощью соответствующих оценок слабого типа.

Запишем операторы $A_{i}$ в виде

$$
A_{i} f(x)=\int_{G} k_{i}(x, y) f(y) d y \quad(i=1,2) .
$$

Воспользуемся результатами работ [13], [16], [17], содержащих весовые оценки слабого и сильного типов для операторов вида

$$
K f(x)=\int_{G} k(x, y) f(y) d y, \quad x \in G,
$$

где $k: G \times G \rightarrow \mathbb{R}^{1}$ - измеримая неотрицательная функция. Положим

$$
k(x, y, R)=\left[1-\chi\left(\frac{x-y}{R}\right)\right] k(x, y) .
$$

При $1 \leqslant p<q<\infty$ и весовых функциях $v, w$ рассмотрим следующую характеристику ядра $k[13],[16],[17]:$

$$
\left\|k v^{-\frac{1}{p}}\right\|_{p, q, w}=\sup _{x \in G} \sup _{R>0}\left\|\left.k(x, \cdot, R) v(\cdot)^{-\frac{1}{p}}\left|L_{p^{\prime}}(G) \|\right| B(x, R)\right|_{w} ^{\frac{1}{q}} .\right.
$$

Tеорема А [17]. Пусть $1 \leqslant p<q<\infty, v, w: G \rightarrow(0, \infty)$ - весовъце функиии, $K$ - оператор из (4.4) с ядром $k$. Тогда

$$
|K f(x)| \leqslant 8\left(\frac{1}{p}-\frac{1}{q}\right)^{-\frac{p}{q}}\left\|k v^{-\frac{1}{p}}\left|\left\|_{p, q, w}\right\| f v^{\frac{1}{p}}\right| L_{p}(G)\right\|^{1-\frac{p}{q}} M_{w}\left(|f|^{p} v\right)(x)^{\frac{1}{q}}
$$


СлеДСтвИЕ [13], [16], [17]. В условиях теоремъ А

$\sup _{\lambda>0} \lambda^{q}|\{x \in G:|K f(x)|>\lambda\}|_{w} \leqslant 8^{q} \theta_{n}^{q}\left(\frac{1}{p}-\frac{1}{q}\right)^{-p}\left\|k v^{-\frac{1}{p}}\right\|\left\|_{p, q, w}^{q}\right\| f v^{\frac{1}{p}} \mid L_{p}(G) \|^{q}$,

әде $\theta_{n}$ - некоторая постоянная, зависящая лишь от размерности $n$.

Теорема А установлена в [17] на основании рассуждений из [13], [16], приводящих к утверждению следствия для $G=\mathbb{R}^{n}$.

Установим оценки для ядер $k_{i}$ из (4.3) операторов $A_{i}($ см. $(2.11)-(2.14))$. В дальнейшем будем считать, что $s-n / p+n / q \geqslant 0$.

Имеем

$$
\begin{aligned}
|k(x, y, R)| & =\left[1-\chi\left(\frac{y-x}{R}\right)\right] \int_{0}^{r_{\gamma}(0)} t^{s-1-n} \chi\left(\frac{y-x}{t}\right) d t \\
& \leqslant C \chi_{B\left(x, \delta r_{\gamma}(0)\right) \backslash B(x, R)}(y) \cdot\left\{\begin{array}{l}
|y-x|^{s-n} \text { при } s<n, \\
\ln \frac{r_{\gamma}(0)}{|y-x|} \quad \text { при } s=n, \\
r_{\gamma}(0)^{s-n} \quad \text { при } s>n .
\end{array}\right.
\end{aligned}
$$

Отсюда

$$
\begin{aligned}
& \left\|k_{1}(x, \cdot, R) v(\cdot)^{-\frac{1}{p}} \mid L_{p^{\prime}}(G)\right\| \\
& \leqslant C_{s} \cdot \begin{cases}r_{\gamma}(0)^{(s-n)_{+}} \\
\times\left(\int_{B\left(x, r_{\gamma}(0)\right) \backslash B(x, R)}|y-x|^{(s-n)-p^{\prime}} v(y)^{-\frac{p^{\prime}}{p}} d y\right)^{\frac{1}{p^{\prime}}} \text { при } s \neq n, \\
\left(\int_{B\left(x, r_{\gamma}(0)\right) \backslash B(x, R)} \ln ^{p^{\prime}} \frac{r_{\gamma}(0)}{|y-x|} v(y)^{-\frac{p^{\prime}}{p}} d y\right)^{\frac{1}{p^{\prime}}} & \text { при } s=n .\end{cases}
\end{aligned}
$$

Загрубив (при $s=n$ ) последнюю оценку ради упрощения ее вида, при произвольном $\varepsilon \in\left(0, n / p^{\prime}+n / q\right]$ имеем

$$
\begin{aligned}
& \left\|k_{1}(x, \cdot, R) v(\cdot)^{-\frac{1}{p}} \mid L_{p^{\prime}}(G)\right\| \leqslant C_{s, \varepsilon} r_{\gamma}(0)^{(s-n)_{+}^{(\varepsilon)}} \\
& \quad \times\left(\int_{B\left(x, r_{\gamma}(0)\right) \backslash B(x, R)}|y-x|^{(s-n)_{-}^{(\varepsilon)} p^{\prime}} v(y)^{-\frac{p^{\prime}}{p}} d y\right)^{\frac{1}{p^{\prime}}} .
\end{aligned}
$$

Оценки (4.7), (4.8) упрощаются в случае, когда вес $v$ удовлетворяет следующему условию: сушествуют постоянные $c_{1}>0, c_{2}>0$ такие, что

$$
c_{1} v(x) \leqslant v(y) \leqslant c_{2} v(x) \text { при }|y-x| \leqslant \delta \rho_{1}(x), \quad x \in G .
$$

При вьполнении (4.9) из (4.8) следует, что

$$
\begin{aligned}
& \left\|k_{1}(x, \cdot, R) v(\cdot)^{-\frac{1}{p}} \mid L_{p^{\prime}}(G)\right\| \\
& \quad \leqslant C_{s, \varepsilon} v(x)^{-\frac{1}{p}} r_{\gamma}(0)^{(s-n)_{+}^{(\varepsilon)}}\left(\int_{R<|z| \leqslant r_{\gamma}(0)}|z|^{(s-n)_{-}^{(\varepsilon)} p^{\prime}} d z\right)^{\frac{1}{p^{\prime}}}
\end{aligned}
$$


откуда при любом достаточно малом $\varepsilon>0$ имеем

$$
\begin{aligned}
& \left\|k_{1}(x, \cdot, R) v(\cdot)^{-\frac{1}{p}} \mid L_{p^{\prime}}(G)\right\| \\
& \quad \leqslant C_{s, \varepsilon} \chi\left(\frac{R}{r_{\gamma}(0)}\right) v(x)^{-\frac{1}{p}}\left(\frac{r_{\gamma}(0)}{R}\right)^{\left(s-\frac{n}{p}\right)_{+}^{(\varepsilon)}} R^{s-\frac{n}{p}} .
\end{aligned}
$$

При изучении оператора $A_{2}$ в неявной форме будем использовать соображения из замечания 2.1. Напомним, что оператор $A_{2}$ построен по правильньм усеченньпм гибким конусам $\left(\gamma, r_{\gamma}\right)$. Для ядра $k_{2}$ справедлива при $1 \leqslant p<\infty$ оценка

$$
\begin{aligned}
& \left\|k_{2}(x, \cdot, R) v(\cdot)^{-\frac{1}{p}} \mid L_{p^{\prime}}(G)\right\| \\
& \quad \leqslant C_{0}^{\frac{1}{p}}\left\|\left(\cdot+r_{\gamma}(0)\right)^{s-1} r_{\gamma}(0)^{\frac{1-n}{p}} V_{p, R}(x, \cdot) \mid L_{p^{\prime}}\left(\left(0, t_{x}\right]\right)\right\|,
\end{aligned}
$$

где

$$
\begin{aligned}
V_{p, R}(x, t) & =r_{\gamma}(t)^{-\frac{n}{p^{\prime}}}\left\|v^{-\frac{1}{p}} \mid L_{p^{\prime}}\left(B\left(\gamma(t), r_{\gamma}(t)\right) \backslash B(x, R)\right)\right\| \\
& =r_{\gamma}(t)^{-\frac{n}{p^{\prime}}}\left(\left|B\left(\gamma(t), r_{\gamma}(t)\right) \backslash B(x, R)\right|_{v^{-\frac{p^{\prime}}{p}}}^{\frac{1}{p^{\prime}}}\right. \\
& =C_{1}\left(\frac{\left|B\left(\gamma(t), r_{\gamma}(t)\right) \backslash B(x, R)\right|_{v^{-\frac{p^{\prime}}{p}}}}{\left|B\left(\gamma(t), r_{\gamma}(t)\right)\right|_{1}}\right)^{\frac{1}{p^{\prime}}} .
\end{aligned}
$$

В самом деле, применяя в случае $p>1$ неравенство Гёльдера по $t$ и затем лемму 3.2 , имеем

$$
\begin{aligned}
\| k_{2}(x, & , R) v(\cdot)^{-\frac{1}{p}} \mid L_{p^{\prime}}(G) \|^{p^{\prime}} \\
= & \int_{G \backslash B(x, R)}\left[\int_{0}^{t_{x}}\left(t+r_{\gamma}(0)\right)^{s-1} r_{\gamma}(t)^{-n} \chi\left(\frac{y-\gamma(t)}{r_{\gamma}(t)}\right) d t\right]^{p^{\prime}} v(y)^{-\frac{p^{\prime}}{p}} d y \\
\leqslant & \int_{G \backslash B(x, R)}\left[\int_{0}^{t_{x}}\left(t+r_{\gamma}(0)\right)^{(s-1) p^{\prime}} r_{\gamma}(t)^{\left(\frac{1}{p}-n\right) p^{\prime}} \chi\left(\frac{y-\gamma(t)}{r_{\gamma}(t)}\right) d t\right] \\
& \times\left[\int_{0}^{t_{x}} r_{\gamma}(t)^{-1} \chi\left(\frac{y-\gamma(t)}{r_{\gamma}(t)}\right) d t\right]^{\frac{p^{\prime}}{p}} v(y)^{-\frac{p^{\prime}}{p}} d y \\
\leqslant & C_{0}^{\frac{p^{\prime}}{p}} \int_{G \backslash B(x, R)} \int_{0}^{t_{x}}\left(t+r_{\gamma}(0)\right)^{(s-1) p^{\prime}} r_{\gamma}(t)^{\left(\frac{1}{p}-n\right) p^{\prime}} \\
& \times \chi\left(\frac{y-\gamma(t)}{r_{\gamma}(t)}\right) d t v(y)^{-\frac{p^{\prime}}{p}} d y,
\end{aligned}
$$

откуда и следует (4.11). 
При выполнении условия (4.9) из (4.10) имеем

$$
\begin{aligned}
& \left\|k_{2}(x, \cdot, R) v(\cdot)^{-\frac{1}{p}} \mid L_{p^{\prime}}(G)\right\| \\
& \leqslant C_{1}\left\|\chi\left(\frac{R}{|\gamma(t)-\gamma(0)|+r_{\gamma}(t)}\right)\left(\cdot+r_{\gamma}(0)\right)^{s-1} r_{\gamma}(\cdot)^{\frac{1-n}{p}} v(\gamma(\cdot))^{-\frac{1}{p}} \mid L_{p^{\prime}}\left(\left(0, t_{x}\right]\right)\right\| .
\end{aligned}
$$

Здесь учтено, что при $y \in B\left(\gamma(t), r_{\gamma}(t)\right) \backslash B(x, R)$

$$
R<|y-x| \leqslant|y-\gamma(t)|+|\gamma(t)-\gamma(0)| \leqslant|\gamma(t)-\gamma(0)|+r_{\gamma}(t) .
$$

Лемма 4.2. Пусть $1 \leqslant p \leqslant \widetilde{q}<q<\infty, s \in \mathbb{N}, s-n / p+n / q \geqslant 0, \delta>0, \varepsilon>0$, $v, w: G \rightarrow(0, \infty)$ - весовые функиии.

Пусть выполнено условие (1.7) или в случае веса v со свойством (4.9) условие

$$
\sup _{x \in G R>0} \sup _{R \rightarrow} \chi\left(\frac{R}{r_{\gamma}(0)}\right) v(x)^{-\frac{1}{p}}\left(\frac{r_{\gamma}(0)}{R}\right)^{\left(s-\frac{n}{p}\right)_{+}^{(\varepsilon)}} R^{s-\frac{n}{p}} \mid G \cap B(x, R)^{\frac{1}{q}}<\infty .
$$

Тогда существует постоянная $C>0$ такая, что для любой функиии $f \in L_{p, v}(G)$

i) $\left|A_{1} f(x)\right| \leqslant C\left\|f v^{\frac{1}{p}} \mid L_{p}(G)\right\|^{1-\frac{p}{q}} M_{w}\left(|f|^{p} v\right)(x)^{\frac{p}{q}}$,

ii) $\sup _{\lambda>0} \lambda\left|\left\{x \in G:\left|A_{1} f(x)\right|>\lambda\right\}\right|_{w}^{\frac{1}{q}} \leqslant C\left\|f v^{\frac{1}{p}} \mid L_{p}(G)\right\|$,

iii) $\left\|A_{1} f\left|L_{\widetilde{q}, w}(G)\left\|\leqslant C N^{\frac{1}{\widetilde{q}}-\frac{1}{q}}\right\| f\right| L_{p, v}(G)\right\|$, əде $N=\sup _{x \in \mathbb{R}^{n}}|G \cap B(x, 1)|_{w}$.

ДокАЗАТЕльство. Из (1.7) или (4.14) следует конечность $\left\|k_{1} v^{-\frac{1}{p}}\right\| \|_{p, q, w}$ в силу оценок (4.8) или (4.10). Тогда утверждения i), іi) вытекают из теоремы А и следствия из нее.

Установим іiі). Пусть $Q_{z}=z+\left(-\frac{1}{2}, \frac{1}{2}\right), z \in \mathbb{Z}$. Заметим, что

$A_{1} f(x)=A_{1} f_{z}(x)$ при $x \in Q_{z}, \quad$ где $f_{z}(x)=\left\{\begin{array}{l}f(x) \text { при } x \in z+\left(-\frac{1}{2 \delta}, \frac{1}{2 \delta}\right), \\ 0 \quad \text { при } x \notin z+\left(-\frac{1}{2 \delta}, \frac{1}{2 \delta}\right) .\end{array}\right.$

Имеем

$$
\begin{aligned}
\left\|A_{1} f \mid L_{\widetilde{q}, w}(G)\right\| & =\left(\sum_{z \in \mathbb{Z}^{n}}\left\|A_{1} f \mid L_{\widetilde{q}, w}\left(G \cap Q_{z}\right)\right\|^{\widetilde{q}}\right)^{\frac{1}{\widetilde{q}}} \\
& \leqslant\left(\sum_{z \in \mathbb{Z}^{n}}\left\|A_{1} f \mid L_{\widetilde{q}, w}\left(G \cap Q_{z}\right)\right\|^{p}\right)^{\frac{1}{p}} \\
& =\left(\sum_{z \in \mathbb{Z}^{n}}\left\|A_{1} f_{z} \mid L_{\widetilde{q}, w}\left(G \cap Q_{z}\right)\right\|^{p}\right)^{\frac{1}{p}} .
\end{aligned}
$$

В случае $\left|G \cap Q_{z}\right|_{w}>0$

$$
\left\|\left.A_{1} f_{z}\left|L_{\widetilde{q}, w}\left(G \cap Q_{z}\right) \|^{\widetilde{q}}=\widetilde{q} \int_{0}^{\infty} \lambda^{\widetilde{q}-1}\right|\left\{x: x \in G \cap Q_{z},\left|A_{1} f_{z}(x)\right|>\lambda\right\}\right|_{w} d \lambda .\right.
$$


Выбирая $\lambda_{0}=C\left\|\left.f_{z} v^{\frac{1}{p}}\left|L_{p}(G) \|\right| G \cap Q_{z}\right|_{w} ^{-\frac{1}{q}}\right.$ и используя утверждение іi), имеем

$$
\begin{aligned}
\left\|A_{1} f_{z} \mid L_{\widetilde{q}, w}\left(G \cap Q_{z}\right)\right\|^{\widetilde{q}} & \leqslant \widetilde{q} \int_{0}^{\lambda_{0}} \lambda^{\widetilde{q}-1}\left|G \cap Q_{z}\right|_{w} d \lambda+\widetilde{q} \int_{\lambda_{0}}^{\infty} \lambda^{\widetilde{q}-1-q} \lambda_{0}^{q}\left|G \cap Q_{z}\right|_{w} d \lambda \\
& =\left|G \cap Q_{z}\right|_{w} \lambda_{0}^{\widetilde{q}}\left(1+\frac{\widetilde{q}}{q-\widetilde{q}}\right) \\
& =\frac{q}{q-\widetilde{q}} C^{\widetilde{q}} N^{\widetilde{q}}\left|G \cap Q_{z}\right|_{w}^{1-\frac{\widetilde{q}}{q}} \|\left. f_{z} v^{\frac{1}{p}}\left|L_{p}(G)\right|\right|^{\widetilde{q}} .
\end{aligned}
$$

Отсюда и из (4.15) получаем, что

$\left\|A_{1} f\left|L_{\widetilde{q}, w}(G)\left\|\leqslant C_{1} N^{\frac{1}{q}-\frac{1}{q}}\left(\sum_{z \in \mathbb{Z}^{n}}\left\|f_{z} v^{\frac{1}{p}} \mid L_{p}(G)\right\|^{p}\right)^{\frac{1}{p}} \leqslant C_{2} N^{\frac{1}{q}-\frac{1}{q}}\right\| f v^{\frac{1}{p}}\right| L_{p}(G)\right\|$.

Из леммы 4.2 в случае $v(x)=\rho_{1}(x)^{a}, w(x)=\rho_{1}(x)^{b}$ вытекает

СлЕДСТВИЕ 4.1. Пусть $s \in \mathbb{N}, 1 \leqslant p<q<\infty, s-n / p+n / q \geqslant 0, v(x)=$ $\rho_{1}(x)^{a}, w(x)=\rho_{1}(x)^{b}, a \in(-\infty, \infty), b \geqslant 0$.

Тогда при $r_{\gamma}(t)=\delta \rho_{1}(\gamma(t))$ выполняются утвержсдения і), ii) леммы 4.2.

ДокАЗАТЕЛЬСТво. В силу (1.1) при $|y-x| \leqslant \delta \rho_{1}(x)$

$$
\left|\rho_{1}(y)-\rho_{1}(x)\right| \leqslant|y-x| \leqslant \delta \rho_{1}(x),
$$

так что

$$
(1-\delta) \rho_{1}(x) \leqslant \rho_{1}(y) \leqslant(1+\delta) \rho_{1}(x)
$$

и условие (4.9) выполнено.

Для выполнения условия (4.14) достаточно, чтобы

$$
\begin{gathered}
\sup _{x \in G} \sup _{0<R \leqslant \delta \rho_{1}(x)} \rho_{1}(x)^{-\frac{a}{p}}\left(\frac{\rho_{1}(x)}{R}\right)^{\left(s-\frac{n}{p}\right)^{(\varepsilon)}} R^{s-\frac{n}{p}} \rho_{1}(x)^{\frac{b}{q}} R^{\frac{n}{q}} \\
\leqslant C \sup _{x \in G} \rho_{1}(x)^{-\frac{a}{p}+s-\frac{n}{p}+\frac{n}{q}+\frac{b}{q}} \leqslant C_{1},
\end{gathered}
$$

что в условиях следствия имеет место. Остается воспользоваться леммой 4.2.

Лемма 4.3. Пусть $1 \leqslant p \leqslant \widetilde{q}<q<\infty, s \in \mathbb{N}, s-n / p+n / q \geqslant 0, \delta>0, \varepsilon>0$, $v, w: G \rightarrow(0, \infty)$ - весовъе функиии и выполняется условие (1.8) или в случае веса $v$ со свойством (4.9) условие

$$
\begin{aligned}
\sup _{x \in G} \sup _{R>0}\left\{\int_{0}^{t_{x}}\right. & \chi\left(\frac{R}{|\gamma(t)-\gamma(0)|+r_{\gamma}(t)}\right)\left(t+r_{\gamma}(0)\right)^{(s-1) p^{\prime}} \\
& \left.\times r_{\gamma}(t)^{\frac{1-n}{p} p^{\prime}} v(\gamma(t))^{-\frac{p^{\prime}}{p}} d t\right\}^{\frac{1}{p^{\prime}}}|B(x, R)|_{w}^{\frac{1}{q}}<\infty .
\end{aligned}
$$

Тогда существует постоянная $C>0$ такая, что для любой функции $f \in L_{p, v}(G)$

i) $\left|A_{2} f(x)\right| \leqslant C\left\|f v^{\frac{1}{p}} \mid L_{p}(G)\right\|^{1-\frac{p}{q}} M_{w}\left(|f|^{p} v\right)(x)^{\frac{p}{q}}$,

ii) $\sup _{\lambda>0} \lambda\left|\left\{x \in G:\left|A_{2} f(x)\right|>\lambda\right\}\right|_{w}^{\frac{1}{q}} \leqslant C N\left\|f v^{\frac{1}{p}} \mid L_{p}(G)\right\| \forall f \in L_{p, v}(G)$,

iii) $\left\|A_{2} f\left|L_{\widetilde{q}, w}(G)\left\|\leqslant C N^{\frac{1}{q}-\frac{1}{q}}\right\| f\right| L_{p, v}(G)\right\|$,

əде $N=\sup _{x \in \mathbb{R}^{n}}|G \cap B(x, 1)|_{w}$. 
ДокАЗАТЕЛЬСтво. Из (1.8) или (4.16) следует конечность $\left\|k_{2} v^{-\frac{1}{p}}\right\| \|_{p, q, w}$ в силу оценок (4.11), (4.13). Тогда утверждения i), ii) вытекают из теоремы А и следствия из нее.

Утверждение іiі) устанавливается так же, как утверждение іiі) леммы 4.2.

СлЕДСТВИЕ 4.2. Пусть $G$ - область с условием гибкого б-конуса $(\sigma \geqslant 1)$, $s \in \mathbb{N}, 1 \leqslant p<q<\infty, v(x)=\rho_{1}(x)^{a}, w(x)=\rho_{1}(x)^{b}, a \in(-\infty, \infty), b \geqslant 0$,

$$
s-\frac{n+a}{p}+\frac{n+b}{q} \geqslant 0, \quad s-\frac{\sigma(n-1)+1+\sigma a}{p}+\frac{n+b}{q} \geqslant 0 .
$$

Тогда выполняются утверждения і), іi) леммы 4.3.

ДокАЗАТЕльство. Поскольку $v(x)=\rho_{1}(x)^{a}$ удовлетворяет условию (4.9), достаточно показать, что выполняется условие (4.16) при $t_{x}=t^{*}, 2 r_{\gamma}(t)=$ $\widetilde{\rho}_{1}(\gamma(t)) \geqslant 2 t^{\sigma}\left(t \in\left(0, t^{*}\right]\right)$.

Для этого достаточно показать, что

$$
\sup _{x \in G} \sup _{R>0}\left[I_{1}(x, R)+I_{2}(x, R)\right]<\infty,
$$

где

$$
\begin{gathered}
I_{1}(x, R)=\left\{\int_{0}^{\tau_{x}} \chi\left(\frac{R}{3 \rho_{1}(x)}\right) \rho_{1}(x)^{\left(s-1+\frac{1-n-a}{p}\right) p^{\prime}} d t\right\}^{\frac{1}{p^{\prime}}}\left(\rho_{1}(x)+R\right)^{\frac{b}{q}} R^{\frac{n}{q}}, \\
I_{2}(x, R)=\left\{\int_{\tau_{x}}^{t^{*}} \chi\left(\frac{R}{3 C_{0} t}\right) t^{(s-1) p^{\prime}} t^{\sigma \frac{1-n-a}{p} p^{\prime}} d t\right\}^{\frac{1}{p^{\prime}}}\left(\rho_{1}(x)+R\right)^{\frac{b}{q}} R^{\frac{n}{q}}, \\
\tau_{x}=\min \left\{t^{*}, \frac{1}{C_{0}} \rho_{1}(x)\right\},
\end{gathered}
$$

$C_{0}$ взято из (1.4). Но

$$
\begin{aligned}
& I_{1}(x, R) \leqslant C_{1} \chi\left(\frac{R}{3 \rho_{1}(x)}\right) \rho_{1}(x)^{s-\frac{n+a}{p}+\frac{n+b}{q}} \leqslant C_{1}<\infty, \\
& I_{2}(x, R)=0 \text { при } \frac{1}{C_{0}} \rho_{1}(x) \geqslant t^{*},
\end{aligned}
$$

а при $\tau_{x}=\frac{1}{C_{0}} \rho_{1}(x)<t^{*}$ имеем следуюшее.

В случае $s-\frac{\sigma(n-1)+1+\sigma a}{p}<0$

$$
I_{2}(x, R) \leqslant C \chi\left(\frac{R}{3 C_{0} t^{*}}\right)\left(\rho_{1}(x)+R\right)^{s-\frac{\sigma(n-1)+1+\sigma a}{p}+\frac{b}{q}} R^{\frac{n}{q}} \leqslant C_{1} .
$$

В случае $s-\frac{\sigma(n-1)+1+\sigma a}{p}>0$

$$
I_{2}(x, R) \leqslant C \chi\left(\frac{R}{3 C_{0} t^{*}}\right)\left(t^{*}\right)^{s-\frac{\sigma(n-1)+1+\sigma a}{p}+\frac{n+b}{q}} \leqslant C_{2} .
$$

В случае $s-\frac{\sigma(n-1)+1+\sigma a}{p}=0$

$$
I_{2}(x, R) \leqslant C \chi\left(\frac{R}{3 C_{0} t^{*}}\right)\left(\ln \frac{3 C_{0} t^{*}}{R}\right)^{\frac{1}{p^{\prime}}}\left(t^{*}\right)^{\frac{b}{q}} R^{\frac{n}{q}} \leqslant C_{3} .
$$

Из оценок, полученных для $I_{1}(x, R), I_{2}(x, R)$, вытекает (4.17) и утверждение следствия. 
TeOpema B. Пусть $1 \leqslant p, q<\infty, 1 \leqslant p_{1}<q_{1}<\infty, 1 / p_{0}=1 / p_{1}-1 / q_{1}+1 / q$, $v, w: G \rightarrow(0, \infty)$ - весовъе функции, $K$ - оператор (4.4) $с$ ядром $k$.

Тогда для $x \in G$

$$
\begin{aligned}
&|K f(x)|<2 \cdot 4^{\frac{p_{0}}{q}}\left(\frac{1}{p_{1}}-\frac{1}{q_{1}}\right)^{-\frac{p_{0}}{q}}\left\|k v^{-\frac{1}{p}}\right\|_{p, q, w}^{1-\frac{p}{q}} \cdot\left\|k v^{-\frac{1}{p}}\right\|_{p_{1}, q_{1}, w}^{\frac{p_{1}}{q_{1}}} \\
& \times\left\|f v^{\frac{1}{p}} \mid L_{p}(G)\right\|^{p_{0}\left(\frac{1}{p_{1}}-\frac{1}{q_{1}}\right)}\left(M_{w}\left(|f|^{p_{1}} v^{\frac{p_{1}}{p}}\right)(x)\right)^{\frac{p_{0}}{p_{1} q}} .
\end{aligned}
$$

Если же $п$ жи $\quad w=1$ дополнительно $q_{1}<q, \quad\left\|k v^{-\frac{1}{p}}\right\|_{p, q, 1}<\infty$, $\left\|k v^{-\frac{1}{p}}\right\|_{p_{1}, q_{1}, 1}<\infty$, то существует постоянная $C>0$, при которой

$$
\left\|K f\left|L_{q}(G)\|<C\| f\right| L_{p, v}(G)\right\| .
$$

ДоказАТельство. Оценка (4.18) в случае $p_{0}=p$ приведена в [17]. В обшем случае доказательство то же. Для доказательства (4.19) возведем в степень $q$ обе части неравенства (4.18) и проинтегрируем по $G$. Учитывая, что $r_{0}=$ $p_{0} / p_{1}>1$, и используя ограниченность максимального оператора $M_{1}$ в $L_{r_{0}}(G)$, получаем (4.19).

Teорема С. Пусть $1 \leqslant p<q<\infty, v, w: G \rightarrow(0, \infty)$ - весовъе функиии, $K$ - оператор (4.4) с ядром $k$.

Пусть $\sum_{-\infty}^{\infty} \zeta_{j}<\infty$, әде

$$
\zeta_{j}=\sup _{x \in G}\left\|\left.k\left(x, \cdot, 2^{-j-1}\right) v(\cdot)^{-\frac{1}{p}}\left|L_{p^{\prime}}(G) \|\right| G \cap B\left(x, 2^{-j}\right)\right|_{w} ^{\frac{1}{q}} .\right.
$$

Тогда существует постоянная $C>0$, при которой имеет место неравенство

$$
\left\|K f\left|L_{q, w}(G)\|\leqslant C\| f\right| L_{p, v}(G)\right\|
$$

для всех функиий $f$ с конечной правой частью.

ДокАЗАТЕЛЬСТво. Достаточно, очевидно, считать $v=1$. Положим

$$
g_{R}(x)=\frac{1}{|G \cap B(x, R)|_{w}} \int_{G \cap B(x, R)}|g(y)| d y .
$$

Представляя интеграл в (4.4) в виде суммы ряда интегралов по $G \cap\left(B\left(x, 2^{-j}\right) \backslash\right.$ $\left.B\left(x, 2^{-j-1}\right)\right)$ и применяя неравенство Гёльдера, имеем

$$
\begin{aligned}
|K f(x)| & \leqslant \sum_{-\infty}^{\infty}\left\|k(x, \cdot)\left|L_{p^{\prime}}\left(G \backslash B\left(x, 2^{-j-1}\right)\right)\|\| f\right| L_{p}\left(G \cap B\left(x, 2^{-j}\right)\right)\right\| \\
& \leqslant\left.\sum_{-\infty}^{\infty} \zeta_{j}\left|G \cap B\left(x, 2^{-j}\right)\right|\right|_{w} ^{-\frac{1}{q}}\left\|f\left|L_{p}(G)\left\|^{1-\frac{p}{q}}\right\| f\right| L_{p}\left(G \cap B\left(x, 2^{-j}\right)\right)\right\|^{\frac{p}{q}} \\
& =\left\|f \mid L_{p}(G)\right\|^{1-\frac{p}{q}} \sum_{-\infty}^{\infty} \zeta_{j}\left(\left(|f|^{p}\right)_{2^{-j}}(x)\right)^{\frac{1}{q}}
\end{aligned}
$$


С помощюю неравенства Гёльдера для последовательностей получаем отсюда оценкy

$$
|K f(x)|^{q} \leqslant C\left\|f \mid L_{p}(G)\right\|^{q-p} \sum_{-\infty}^{\infty} \zeta_{j}\left(|f|^{p}\right)_{2^{-j}}(x)
$$

откуда

$$
\left\|K f\left|L_{q, w}(G)\left\|^{q} \leqslant C\right\| f\right| L_{p}(G)\right\|^{q-p}\left(\sum_{-\infty}^{\infty} \zeta_{j}\right)^{q} \sup _{j}\left\|\left(|f|^{p}\right)_{2^{-j}} \mid L_{1}(G)\right\| .
$$

Теперь для завершения доказательства теоремы достаточно воспользоваться следуюшей леммой.

Лемма 4.4. Существует постоянная $C>0$, при которой

$$
\left\|g_{R}\left|L_{1, w}(G)\|\leqslant C\| g\right| L_{1}(G)\right\|
$$

для всех открытых мнохсеств $G \subset \mathbb{R}^{n}$, всех $R>0$ и всех $g \in L_{1}(G)$.

ДокАЗАтЕльство. Будем считать, что $G$ - ограниченное множество. Доказательство (4.20) для неограниченного множества $G$ следует из $(4.20)$ для $G \cap B(x, N)$ (в качестве $G$ ) с помощью предельного перехода при $N \rightarrow \infty$. Введем

$$
E_{m}=\left\{x \in G: 2^{-m}<g_{R}(x) \leqslant 2^{-m+1}\right\}, \quad m \in \mathbb{Z} .
$$

Рассмотрим сначала случай, когда $g_{R}$ - ограниченная функция. Тогда при некотором $m_{1} \in \mathbb{Z} E_{m_{1}} \neq \varnothing, E_{j}=\varnothing$ при $j<m_{1}$.

Построим последовательность $\left\{B_{k}\right\}$ шаров $B_{k}=B\left(x^{(k)}, R\right)$ следующим образом. Возьмем $x^{(1)} \in E_{m_{1}}$. Если $x^{(1)}, \ldots, x^{(k)}$ уже выбраны, выберем $x^{(k+1)} \in G \backslash$ $\bigcup_{1 \leqslant j \leqslant k} B_{j}, x^{(k+1)} \in E_{m_{k+1}}$, где $m_{k+1}=\min \left\{s: s \geqslant m_{1}, E_{s} \backslash \bigcup_{1 \leqslant j \leqslant k} B_{j} \neq \varnothing\right\}$.

В силу ограниченности $G$ процесс оборвется после некоторого шага, и требуемая (конечная) последовательность построена.

Положив $D_{k}=\left(G \cap B_{k}\right) \backslash \bigcup_{1 \leqslant j \leqslant k-1} B_{j}$ при $1 \leqslant k \leqslant N$, отметим, что $D_{j} \cap$ $D_{k}=\varnothing$ при $j \neq k, G=\bigcup_{1 \leqslant k \leqslant N} D_{k}$,

$$
g_{R}(x) \leqslant 2 g_{R}\left(x^{(k)}\right) \text { при } x \in D_{k} .
$$

Поэтому

$$
\int_{G} g_{R}(x) d w \leqslant \sum_{k=1}^{N} 2 \int_{D_{k}} g_{R}\left(x^{(k)}\right) d w \leqslant 2 \sum_{k=1}^{N} \int_{G \cap B_{k}}|g(y)| d y .
$$

Но кратность пересечения $\left\{B_{k}\right\}_{1}^{N}$ не превосходит, очевидно, некоторого числа $\varkappa_{n}$, зависящего лишш от размерности $n$. Поэтому из последней цепочки неравенств получаем (4.20) с $C=2 \varkappa_{n}$ при условии, что функция $g_{R}$ ограничена.

Пусть теперь $g_{R}$ неограничена. Тогда с помощью приведенных рассуждений можно показать, что

$\left\|g_{R}\left|L_{1, w}\left(G^{(T)}\right)\left\|\leqslant 2 \varkappa_{n}\right\| g\right| L_{1}(G)\right\|, \quad$ где $T>0, \quad G^{(T)}=\left\{x \in G: g_{R}(x)<T\right\}$.

Переходя в последнем неравенстве к пределу при $T \rightarrow \infty$, получаем (4.20). Лемма доказана. 


\section{§5. Доказательство основных результатов}

В силу леммы 3.1 везде в $\oint 5$ будем считать, что $\left(G, \gamma, r_{\gamma}\right) \in \mathscr{G}_{0}\left(\delta, R_{0}, C_{0}\right)$.

ДокаЗАТЕЛЬство ЛЕммы 1. Воспользуемся оценкой (2.11). Первые два слагаемых ее правой части оцениваются с помощью утверждений і) соответственно лемм 4.2, 4.3, а третье слагаемое - с помощью леммы 4.1.

ДОКАЗАТЕЛЬСТво ТЕОРЕМЫ 1 следует в силу (2.11) из утверждений іi) лемм $4.2,4.3$ и леммы 4.1 .

ДОКАЗАТЕЛЬСТВО ТЕОРЕМЫ 2 в случае $p<\widetilde{q}<q$ следует в силу (2.11) из утверждений іiі) лемм 4.2, 4.3 и леммы 4.1 .

В случае $s=1, p<\widetilde{q}=q$ применим метод срезки функции по уровням (truncated method), предложенный В.Г. Мазьей при оценке интеграла, содержащего емкость (1964 г., см. [2; п. 2.3.1]). Этот метод использовался в [10] для получения оценки сильного типа из оценки слабого типа. Здесь мы применяем иной вариант рассуждений, которьй также основан на учете распределения значений функции по уровням. Пусть $\varphi \in C_{0}^{\infty}\left(\mathbb{R}^{1}\right), \operatorname{supp} \varphi \subset\left(\frac{1}{2}, 2\right), \varphi_{k}(t)=\varphi\left(2^{-k} t\right), \sum_{-\infty}^{\infty} \varphi_{k}(t)=1$ при $t>0$. Пусть $f: G \rightarrow \mathbb{R}$ имеет обобшенные по Соболеву производные первого порядка и конечна правая часть (3) при $s=1$. Положим $f_{k}(x)=\varphi\left(2^{-k}|f(x)|\right) f(x)$. Тогда $f(x)=\sum_{-\infty}^{\infty} f_{k}(x)$, причем для каждого $x \in G$ не более двух членов ряда отличны от нуля.

Заметим также, что

$$
D_{i} f_{k}(x)=\varphi^{\prime}\left(2^{-k}|f(x)|\right) 2^{-k}(\operatorname{sgn} f(x)) f(x) D_{i} f(x)+\varphi\left(2^{-k}|f(x)|\right) D_{i} f(x) .
$$

Положим

$$
E_{k}=\left\{x \in G:|f(x)|>2^{k}\right\}, \quad E_{k}^{*}=\left\{x \in G:\left|f_{k}(x)\right|>2^{k-2}\right\} .
$$

Тогда

$$
\begin{aligned}
I^{q} & =\int_{G}|f(x)|^{q} w(x) d x=\sum_{k \in \mathbb{Z}} \int_{E_{k} \backslash E_{k+1}}|f(x)|^{q} w(x) d x \\
& =\sum_{k} \int_{E_{k} \backslash E_{k+1}}\left|f_{k}(x)+f_{k+1}(x)\right|^{q} w(x) d x \\
& \leqslant \sum_{k} 2^{(k+1) q}\left|\left\{x \in G:\left|f_{k}(x)+f_{k+1}(x)\right|>2^{k}\right\}\right|_{w} \\
& \leqslant \sum_{k} 2^{(k+1) q}\left(\left|E_{k}^{*}\right|_{w}+\left|E_{k+1}^{*}\right| w\right)=2^{2 q}\left(2^{q}+1\right) \sum_{k} 2^{(k-2) q}\left|E_{k}^{*}\right|_{w} .
\end{aligned}
$$

Используя теорему 1 (ее оценку $(4)$ при $s=1$ ), имеем отсюда

$$
\begin{aligned}
I^{q} & \leqslant C \sum_{k}\left(\sum_{i=1}^{n}\left\|D_{i} f_{k}\left|L_{p, v}(G)\|+\| f_{k}\right| L_{r, u}\left(G_{\delta}\right)\right\|\right)^{q} \\
& \leqslant C_{1} \sum_{i=1}^{n}\left(\sum_{k}\left\|D_{i} f_{k} \mid L_{p, v}(G)\right\|^{p}\right)^{\frac{q}{p}}+C_{1}\left(\sum_{k}\left\|f_{k} \mid L_{r, u}\left(G_{\delta}\right)\right\|^{r}\right)^{\frac{q}{r}} \\
& \leqslant C_{2} \sum_{i=1}^{n}\left\|D_{i} f\left|L_{p, v}(G)\left\|^{q}+C_{2}\right\| f\right| L_{r, u}\left(G_{\delta}\right)\right\|^{q}
\end{aligned}
$$

и доказательство теоремы в случае $s=1$ завершено. 
ДоКАЗАТЕЛЬСТво тЕОРЕМЫ 3 . Теорема 3 представляет собой частный случай теоремы 2.

ДОКАЗАТЕЛЬСТВО ТЕОРЕМЫ 4. В силу оценки (2.11) достаточно убедиться в ограниченности операторов $A_{1}, A_{2}: L_{p, \rho_{1}^{a}}(G) \rightarrow L_{q, \rho_{1}^{b}}(G)$ (оператор $A_{3}$ ограничен в силу леммы 4.1). В силу следствий 4.1, 4.2 справедливы оценки слабого типа:

$$
\sup _{\lambda>0} \lambda\left|\left\{x \in G:\left|A_{j} g(x)\right|>\lambda\right\}\right|_{\rho_{1}^{b}}^{\frac{1}{q}} \leqslant C\left\|\rho_{1}^{\frac{a}{p}} g \mid L_{p}(G)\right\| \quad(j=1,2) .
$$

Условия теоремы выполняются также при замене в них пары $(p, q)$ на каждую из пар $\left(p_{0}, q_{0}\right),\left(p_{1}, q_{1}\right)$, где $1<p_{0}<p<p_{1}<q_{1}<\infty, p_{0}<q_{0}<q<q_{1}<\infty\left(p_{0}, p_{1}\right.$ близки к $p$, а $q_{0}, q_{1}$ близки к $\left.q\right)$ и

$$
\frac{1}{2 p_{0}}+\frac{1}{2 p_{1}}=\frac{1}{p}, \quad \frac{1}{2 q_{0}}+\frac{1}{2 q_{1}}=\frac{1}{q} .
$$

Тогда в силу интерполяционной теоремы Марцинкевича существует $C>0$ такое, что

$$
\left\|\rho_{1}^{\frac{b}{q}} A_{j} f\left|L_{q}(G)\|\leqslant C\| \rho_{1}^{\frac{a}{p}} f\right| L_{p}(G)\right\| \quad(j=1,2)
$$

для всех $f$ с конечной правой частью. Этим доказана основная часть теоремы 4.

Пусть $\sigma=1$ или $a \geqslant 1-n$. Тогда доказательство основной части теоремы сохраняется при замене строгого неравенства в іi) на нестрогое. При этом само (нестрогое) неравенство іi) является следствием іiі) и может быть опущено.

В случае $a \geqslant 1-n, b=0$ доказательство основной части теоремы сохраняется при замене строгого неравенства i) на нестрогое, а само (нестрогое) неравенство і) является следствием неравенства iii) и может быть опушено. Теорема доказана.

ДОКАЗАТЕЛЬСТВО ТЕОРЕМЫ 5 сводится в силу (2.11) к доказательству ограниченности операторов $A_{1}, A_{2}: L_{p, v}(G) \rightarrow L_{q}(G), A_{3}: L_{r, u}\left(G_{\delta}\right) \rightarrow L_{q}(G)$. Ограниченность последнего установлена в лемме 4.1. Ограниченность операторов $A_{1}, A_{2}$ вытекает из теоремы В.

ДОКАЗАТЕЛЬСТВО ТЕОРЕМЫ 6 сводится в силу $(2.11)$ к доказательству ограниченности операторов $A_{1}, A_{2}: L_{p, v}(G) \rightarrow L_{q, w}(G), A_{3}: L_{r, u}\left(G_{\delta}\right) \rightarrow L_{q, w}(G)$. Ограниченность последнего установлена в лемме 4.1. Ограниченность операторов $A_{1}, A_{2}$, которые можно считать построенньми по правильньм усеченньм гибким конусам, вытекает из теоремы С, условия которой выполняются в силу оценок (4.8) (для $\left.A_{1}\right),(4.11)$ (для $A_{2}$ ).

Результаты этой работы, относящиеся к случаю весов $v=\rho_{1}^{a}, w=\rho_{1}^{b}, u=1$, были доложены на семинаре Института математики университета г. Йены (Германия) в октябре 1999 г. Формулировка основных результатов приведена в [18].

\section{Список литературы}

1. Соболев С. Л. Некоторые применения функционального анализа в математической физике. М.: Наука, 1988.

2. Мазья В. Г. Пространства С. Л. Соболева. Л.: Изд-во ЛГУ, 1985.

3. Решетняк Ю. Г. Интегральные представления функций в областях с негладкой границей // Сиб. матем. журн. 1980. Т. 21. №6. С. 108-116. 
4. Гольдштейн В. М., Решетняк Ю.Г. Введение в теорию функций с обобщенньми производньми и квазиконформные отображения. М.: Наука, 1983.

5. Бесов О. В., Ильин В. П., Никольский С. М. Интегральные представления функций и теоремы вложения. М.: Наука, 1996.

6. Глобенко И. Г. Некоторые вопросы теории вложения для областей с особенностями на границе // Матем. сб. 1962. Т. 57. №2. С. 201-224.

7. Лабутин Д. А. Интегралњноепредставление функций и вложение пространств Соболева на областях с нулевыми углами // Матем. заметки. 1997. Т. 61. № 2. С. 201-219.

8. Smith W., Stegenga D. A. Holder domains and Poincaré domains // Trans. Amer. Math. Soc. 1990. V. 319. P. 67-100.

9. Hajlash P., Koskela P. Isoperimetric inequalities and imbedding theorems in irregular domains // J. London Math. Soc. (2). 1998. V. 58. № 185. P. 425-450.

10. Kilpeläinen T., Malý J. Sobolev inequalities on sets with irregular boundaries // Z. Anal. Anwendungen. 2000. V. 19. № 2. P. 369-380.

11. Лабутин Д. А. Вложение пространств Соболева на гёльдеровых областях // Труды МИАН. 1999. Т. 227. С. 170-179.

12. Stredulinsky E. W. Weighted inequalities and degenerate elliptic partial differential equations. Berlin: Springer-Verlag, 1984. (Lecture Notes in Math. V. 1074.)

13. Кокилашвили В. М., Габидзашвили M. A. О весовых неравенствах для анизотропных потенциалов и целых функций // Докл. АН СССР. 1985. Т. 282. №6. С. 1304-1306.

14. Стейн И. Сингулярные интегралы и дифференциальные свойства функций. М.: Мир, 1973.

15. Лабутин Д. А. Неулучшаемость неравенств Соболева для класса нерегулярных областей // Труды МИАН. 2001. Т. 232. С. 218-222.

16. Габидзашвили M. А. Весовые неравенства для анизотропных потенциалов // Труды Тбилисского матем. института. 1986. Т. 82. С. 25-36.

17. Бесов О.В. Вложения пространств дифференцируемых функций переменной гладкости // Труды МИАН. 1997. Т. 214. С. 25-58.

18. Бесов О. В. Теорема вложения Соболева для области с нерегулярной границей // Докл. PAH. 2000. T. 373. № 2. C. 131-134. 